\title{
Schwarzes Mittelmeer, weißes Europa
}

\section{Kolonialität, Rassismus und die Grenzen der Demokratie}

\author{
Black Mediterranean, white Europe \\ Coloniality, Racism, and the Limits of Democracy
}

\section{Jeanette Ehrmann, Giessen}

Zusammenfassung: Die Passage über das Mittelmeer ist in den letzten Jahren zu einer der tödlichsten Migrationsrouten der Welt geworden. Während die Mitgliedsstaaten der Europäischen Union gegen die sogenannte „Flüchtlingskrise“ eine militärische und diskursive Fluchtabwehrpolitik betreiben und die Seenotrettung geflüchteter Menschen aussetzen und kriminalisieren, begreifen normative politische Theorien der Migration Fluchtbewegungen als ein politisches oder moralisches Problem sowie als Krise für etablierte Demokratien. Gegen den Topos der „Flüchtlingskrise" und die implizite Normalisierung von Grenzen in einem Großteil gegenwärtiger politiktheoretischer Debatten zu Migration und Flucht entwickelt der vorliegende Beitrag eine politische Theorie der Grenze aus der Perspektive postkolonialer und Schwarzer kritischer Theorie. Dazu werden zunächst Kolonialität und Rasse als bislang vernachlässigte analytische Kategorien in die politische Theorie eingeführt, um die koloniale Gegenwart von Grenzregimen und die Rassifizierung von Grenzen zu rekonstruieren. Im Rückgriff auf die Figur des Schwarzen Mittelmeers, die aus der radikalen Schwarzen Tradition stammt und im Kontext gegenwärtiger Migrationsrouten von Afrika nach Europa aktualisiert wird, wird sodann ein alternatives Register für eine politische Theorie der Grenze entwickelt: Erstens wird das europäische Grenzregime als eine rassistische Politik der Vergrenzung verstanden; zweitens werden die postkolonialen Ambivalenzen und rassifizierten Dimensionen einer Politik der Solidarität mit geflüchteten Menschen problematisiert; drittens werden Fluchtbewegungen als widerständige politische Imaginationen und Praktiken nachgezeichnet, die die Widersprüche hegemonialer Konzeptionen von Demokratie und Zugehörigkeit aufzeigen. Abschließend wird die Epistemologie des Schwarzen Mittelmeers für eine Kritik des dominanten Krisennarrativs der Demokratie und für eine 
selbstreflexive Auseinandersetzung mit den kognitiven Grenzen der politischen Theorie selbst produktiv gemacht. ${ }^{1}$

Schlagwörter: Migration - Grenze - Kolonialität - Rassismus - Demokratie

Abstract: In recent years, the passage across the Mediterranean Sea has become one of the deadliest migration routes in the world. While the member states of the European Union pursue a military and discursive policy against the so-called "refugee crisis" that securitizes borders and even suspends and criminalizes the sea rescue of refugees, normative political theories of migration often depict border crossings as a political or moral problem as well as a crisis for established democracies. Against the trope of the "refugee crisis" and the tacit normalization of borders in much of current political theory debates on migration and refugees, this article develops a political theory of the border from the perspective of postcolonial critique and Black critical thought. It introduces coloniality and race as previously neglected analytical categories into political theory to theorize the colonial present of border regimes and the racialization of borders. It then draws on the Black Mediterranean-an epistemology that originates from the Black radical tradition and is currently used to discuss African migrants' routes to Europe-to develop an alternative register for a political theory of the border. The epistemology of the Black Mediterranean is unfolded in three respects: first, it allows for the European border regime to be reconstructed as a racialized politics of borderization; second, it highlights the postcolonial ambivalences and racialized dimensions of a politics of pity and solidarity with refugees; third, it understands refugee movements as resistant political imaginations and practices that reveal the contradictions of hegemonic conceptions of democracy and belonging. Finally, the article suggests that the epistemology of the Black Mediterranean offers a critique of the dominant crisis-of-democracy narrative while enabling self-reflective interrogation of the cognitive limits of political theory itself.

Keywords: Migration - Borders - Coloniality - Racism - Democracy

1 Für die wertschätzenden und konstruktiven Diskussionen, Kommentare und Rückfragen, die die Entstehung dieses Beitrags begleitet und bereichert haben, danke ich den Herausgebern des Schwerpunktes, Florian Grosser und Andreas Oberprantacher, den beiden anonymen Gutachter*innen, den Organisator*innen und Teilnehmer*innen des Workshops „Democracy, Critique, and Europe's ,Crisis': Black Feminist and Decolonial Troubles" am Critical Theory Cluster der Northwestern University, insbesondere Penelope Deutscher, Noémi Michel, Barnor Hesse, William Paris und Usdin L. Martínez, sowie Martin Oppelt und den Teilnehmer*innen des Kolloquiums für Politische Theorie an der Hochschule für Politik München. Ein besonderer Dank gilt Angelika Miklin für das umsichtige Lektorat. 


\section{Von Lifeboat Ethics und Left-to-die Boats: Das Schweigen der politischen Theorie}

Der Philosoph Garrett Hardin prägte 1974 die Metapher des Rettungsboots. Sie sollte Gedankenexperimente zu grenzüberschreitender Migration unter Bedingungen globaler Ungleichheit erhellen. Als zentrales Problem einer „Ethik des Rettungsbootes“ konstruierte er folgendes Szenario: „Metaphorically, each rich nation amounts to a lifeboat full of comparatively rich people. The poor of the world are in other, much more crowded lifeboats. Continuously, so to speak, the poor fall out of their lifeboats and swim for a while in the water outside, hoping to be admitted to a rich lifeboat, or in some other way to benefit from the goodies' on board. What should the passengers on a rich lifeboat do?" (Hardin 1974, 561) Im Gegensatz zu einem von Gerechtigkeitsüberlegungen motivierten Idealismus, der letztlich im Ertrinken aller ende, erlaube die Metapher des Rettungsboots ein rationales Abwägen von Rechten und Pflichten, ein sachliches Berechnen von Aufnahmekapazitäten und Ressourcen. Die von Hardin metaphorisch in Szene gesetzte Katastrophe des Schiffbruchs spielt sich in den letzten Jahren vor den Augen der europäischen Öffentlichkeit an den Grenzen Europas ab. Die Passage auf dem Mittelmeer, einer der am intensivsten überwachten Meereszonen der Welt (Heller, Pezzani und Situ Research 2014, 640), ist zur tödlichsten Migrationsroute auf dem Seeweg geworden (United Nations High Commissioner for Refugees 2018). ${ }^{2}$ Auf der zentralen Mittelmeerroute zwischen Libyen, Malta und Italien stirbt fast jede*r sechste Migrant*in auf einem „left-to-die boat“, den namenlosen Booten, die von der libyschen Küste abfahren und keinen sicheren Hafen mehr erreichen.

Die Bezeichnung „left-to-die boat“ geht auf einen Präzedenzfall im Jahr 2011 zurück, bei dem ein Boot mit zweiundsiebzig Menschen aus Nigeria, Ghana, dem Sudan, Eritrea und Äthiopien unter Beobachtung des in Rom ansässigen internationalen Maritime Rescue Coordination Centre, des maltesischen Seenotrettungsdienstes, des Allied Command Ope-

$2 \quad$ Seit 2014 sind dem Missing Migrants Project der Internationalen Organisation für Migration zufolge 20.056 Menschen im Mittelmeer zu Tode gekommen, und dies sind nur die dokumentierten Todesfälle, die statistisch nach Todeszeitpunkt, Todesursache und Herkunftsregion der ertrunkenen Person erfasst werden (IOM 2019). Der überwiegende Großteil der ertrunkenen Menschen stammt aus afrikanischen Ländern südlich der Sahara. Zur Problematik von Statistiken im Kontext eines nekropolitischen Grenzregimes siehe weiter unten. 
rations Headquarter der NATO in Neapel, eines Militärschiffs und eines französischen Militärhubschraubers, der Trinkwasser und Kekse für die Bootsinsassen abwarf, so lange auf dem Mittelmeer trieb, bis es wieder an der libyschen Küste strandete. Einundsechzig Menschen waren zu diesem Zeitpunkt bereits gestorben. Von den elf Überlebenden starben kurze Zeit später zwei weitere Menschen. Die Überlebenden wurden von den libyschen Behörden festgenommen und ohne medizinische Versorgung in ein Lager eingesperrt. Die unterlassene Hilfeleistung trotz Notrufen der Bootsinsassen und genauer Kenntnis der Position des Bootes wurde von der Forschungsgruppe Forensic Oceanography unter Einsatz modernster Technologien rekonstruiert, deren eigentlicher Zweck die Polizierung des Mittelmeers ist (Heller, Pezzani und Situ Research 2014). Entgegen der vielzitierten Trope des Schiffsunglücks ist der minutiöse Bericht das erschütternde Dokument einer bewussten und beobachteten Politik des Ertrinkenlassens.

Wie konnten die Vertreter*innen der Institutionen, die für Seenotrettung zuständig sind, die Menschen hilflos im Wasser treiben lassen? Wie konnte eine solch eklatante Verletzung grundlegender Menschenrechte bis hin zum Recht auf Leben in den Hoheitsgewässern der Europäischen Union (EU) geschehen, deren Gründungs- und Legitimationsnarrativ die moralische Verpflichtung zur Achtung universeller Menschenrechte ist? Diese Frage wurde zum Gegenstand einer von der parlamentarischen Versammlung des Europarates angestrengten Untersuchung. Sie kam zum Ergebnis, dass es sich um ein kollektives Versagen handle, ohne konkrete Verantwortlichkeiten oder Verpflichtungen an die beteiligten Akteure zuzuweisen. Mit dem rechtlich folgenlosen Begriff des Versagens, der unverbindlichen Selbstverpflichtung, aus dem Fall für die Zukunft zu lernen, und der Schuldzuweisung an Menschenschmuggler*innen blieb die Untersuchung praktisch folgenlos (Follis 2015, 51-55). Unbeantwortet blieb auch die im Raum stehende ethische Frage, wessen Überleben auf dem Spiel steht und ob das Recht auf Leben und damit zumindest die Seenotrettung tatsächlich allen Menschen zusteht oder nur Bürger*innen reicher Staaten (Follis 2015, 56). Wie wenig die Menschenleben und die Menschenrechte der Geflüchteten der EU wert sind, wurde schließlich in der verabschiedeten Resolution deutlich, die zwar die traumatischen Folgen des Erlebten anerkennt, dabei aber nicht mögliche Rechtsansprüche der Überlebenden und der Hinterbliebenen klärt. Was als kollektives Versagen verharmlost wurde, kann daher treffender als „death by policy“ bezeichnet werden (UNITED Against Refugee Deaths 2019), als eine bewusste „Politik des Sterbenlassens“ (Hess 2018). 
Während dieser Beitrag geschrieben wird, setzt sich die Arithmetik des Sterbenlassens an den Grenzen Europas täglich fort, werden Menschen statistisch als namen- und gesichtslose Nummern erfasst. Die Überlebenden, die von der libyschen Küstenwache aufgegriffen werden, müssen befürchten, mit Unterstützung der EU in eines der Lager verschleppt zu werden, wo aktuellen Studien zufolge systematisch Folter und sexualisierte Gewalt gegen Mädchen, Frauen, Jungen und Männer eingesetzt werden (Chynoweth 2019). Obwohl die Vereinten Nationen und deren Hochkommissariat für Flüchtlinge (UNHCR) eindringlich an die EU appellieren, die Seenotrettung wieder aufzunehmen und die Auslieferung von Migrant*innen in libysche Lager zu unterbinden, wird die zivile Seenotrettung kriminalisiert und unter Federführung der europäischen Grenzschutzagentur Frontex eine zunehmende Militarisierung der EU-Außengrenzen betrieben (Buckel 2018). ${ }^{3}$ Dass in Europa inzwischen nicht nur das Recht auf Asyl, sondern auch das Recht auf Leben flüchtender Menschen unverhohlen zur Disposition steht, zeigte sich paradigmatisch in einem „Pro und Contra“ der ZEIT zur Legitimität der privaten Seenotrettung mit dem bezeichnenden Titel „Oder soll man es lassen?“ (Lobenstein und Lau 2018). Unterdessen scheitert eine gemeinsame Asylpolitik der EU-Mitgliedsstaaten an der populistisch aufgeheizten „Verteilungsfrage“ (Habermas 2018, 42), die Menschen zu statistischem Material macht.

In einem politischen Moment, in dem unter links- wie rechtspopulistischen Vorzeichen die Nation als Schicksalsgemeinschaft reaktiviert und Migration als Existenzfrage beschworen wird, scheint Hardins Rechenmethode also den Zeitgeist zu treffen (vgl. Slobodian 2019). Nach dem „Sommer der Migration“ im Jahr 2015 und der anfänglichen Euphorie einer „Willkommenskultur“ zumindest in Deutschland setzte sich schon bald der Topos der „Flüchtlingskrise“ durch, der die Figur des „Flüchtlings“ als zentrale Gefahr für die Sicherheit und Souveränität der nationalstaatlich verfassten Demokratie zeichnet. Die Reaktion auf das politisch produzierte und medial inszenierte Krisennarrativ, das Flucht als Krisenerfahrung nicht für geflüchtete Menschen, sondern für die sesshaften Bürger*innen der global betrachtet reichen Aufnahmegesellschaften zeichnet, besteht in einer diskursiven wie

$3 \quad \mathrm{Zu}$ den rechtlichen und politischen Auseinandersetzungen um die Seenotrettung im Mittelmeer seit Anfang der 2000er Jahre bis 2018, der Überwachung der Seeaußengrenzen durch die EU und der Bindung der Grenzschutzagentur Frontex an see- und menschenrechtliche Vorgaben siehe Pichl und Tohidipur (2019). 
militärischen Fluchtabwehrpolitik (Bhagat und Soederberg 2019, 422). Unter Absehung ethischer und menschenrechtlicher Aspekte stehen die ökonomische Frage nach der Aufnahmefähigkeit der EU-Mitgliedsstaaten und die kulturalisierte Frage nach der Integrationsfähigkeit geflüchteter Menschen im Mittelpunkt (Cole 2016, 123-124).

Mit welchen Deutungsangeboten und Lösungsansätzen reagieren politische Theoretiker*innen auf diese politische Konstellation und die mit ihr verbundene populistische Konjunktur? Im Kontrast zum hegemonialen Krisennarrativ konstatiert Gurminder Bhambra angesichts des Ertrinkenlassens im Mittelmeer keine „Flüchtlingskrise“, sondern eine moralische Krise Europas. Besonders irritierend sei das Schweigen der öffentlichen Intellektuellen des Kontinents, vor allem derjenigen, die als prominente Fürsprecher*innen des europäischen Einigungsprojekts aufträten, etwa Jürgen Habermas (Bhambra 2017, 400). Dieses Schweigen führt Bhambra jedoch nicht primär auf ein individuelles moralisches Versagen zurück, sondern vielmehr auf ein konzeptionelles: Es ist das Unvermögen, Europa und vom Standpunkt Europas gedachte Konzeptionen von Demokratie, Staatsbürger*innenschaft und Zugehörigkeit sowie deren Grenzen in ihrer konstitutiven Verflechtung mit einer kolonialen Vergangenheit und einer postkolonialen Gegenwart zu verstehen (ebd.). Auch wenn einzelne politische Theoretiker*innen in den Diskurs um die „Flüchtlingskrise“ intervenieren, fehlt in der politischen Theorie bislang eine grundlegende postkoloniale Kritik von Grenzen. ${ }^{4}$ Eine politische Theorie der Migration, der Flucht und der Grenze bleibt jedoch sowohl analytisch als auch normativ unvollständig, solange gegenwärtige Grenzregime nicht in der „kolonialen Gegenwart“ (Gregory 2004), vor dem Hintergrund der Nachwirkungen des europäischen Kolonialismus verortet werden (so auch Cole 2016, 128). Dies gilt umso mehr für das Mittelmeer, das schon immer ein Raum der zugleich produktiven wie destruktiven kulturellen, politi-

Seyla Benhabib (2015), Nikita Dhawan (2015), Chiara Bottici (2016) und Sonja Buckel (phoenix 2019) kommentieren die „Flüchtlingskrise“ kritisch aus Sicht einer politisch engagierten politischen Theorie und Philosophie. Étienne Balibar, Sandro Mezzadra, Eric Fassin und Barbara Spinelli rufen mit einem internationalen Appell zu Gerechtigkeit, Solidarität und zur Entkriminalisierung der Seenotrettung auf (Balibar et al. 2019). Balibar (2016, 170) betont zudem, dass „das Postkoloniale“ Multikulturalismus und Kosmopolitismus als dominante Konzepte der politischen Theorie zunehmend infrage stellt. Im deutschsprachigen Kontext hat Ina Kerner (2019) eine erste postkoloniale Analyseperspektive für eine politische Theorie der Grenze und der Migration formuliert. 
schen und ökonomischen Begegnung zwischen Europa und Afrika war, einer Begegnung, die unwiderruflich durch die europäische Kolonisierung Afrikas und die Versklavung afrikanischer Menschen belastet ist (Amkpa 2016, 16). Dass Europa als „hero of the colonial adventure“ (Spivak 2013, 100) ein postkolonialer ebenso wie ein Post-Versklavungskontext ist, wird jedoch weder von einer breiteren politischen Öffentlichkeit thematisiert noch in der politischen Theorie systematisch reflektiert.

Entgegen der diskursiven Entortung von Kolonialität jenseits der Grenzen Europas (Gutiérrez Rodríguez 2010, 53) versucht dieser Beitrag eine politische Theorie der Grenze zu skizzieren, die Europa und mit ihr die westeuropäische liberale Demokratie als eine postkoloniale politische Ordnung versteht. Er ist aus der Position der auf sicherem Grund stehenden, mit einer EU-Bürger*innenschaft ausgestatteten Zuschauerin der Katastrophe des Schiffbruchs geschrieben. Im Gegensatz zu Hans Blumenbergs „Schiffbruch mit Zuschauer“ (Blumenberg 1979) ist er dabei nicht von einer unbeteiligten Haltung getragen, sondern von der Einsicht, als „implicated subject“ (Rothberg 2019, 19) in fortwirkende postkoloniale Gewaltformationen verwickelt zu sein, wie sie sich derzeit besonders drastisch im europäischen Grenzregime manifestieren. Die vielfach zirkulierenden Bilder des Schiffbruchs und mehrheitlich Schwarzer schiffbrüchiger Menschen werfen umso dringlicher die Frage der Implikation der Zuschauer*in, der ethischen Dimension ihrer Zeug*innenschaft auf. Als Katastrophenbilder, die die Verletzung von Menschen zeigen, können sie Ariella Azoulay zufolge nicht bloß als ästhetische Artefakte gesehen werden. Sie implizieren vielmehr eine ethische Praxis des Betrachtens, die Azoulay „civic skill“ nennt (Azoulay 2008, 14): Sie legen der mit einer Staatsbürger*innenschaft privilegierten Betrachterin die Verantwortung auf, ihren rechtlich abgesicherten Status nicht als Privateigentum, sondern als Verpflichtung zu begreifen und zu gebrauchen, um Regierungsweisen, der die Betrachterin und die Betrachteten unterworfen sind, neu auszuhandeln. ${ }^{5}$ Wie sich diese von Azoulay als zivile Verant-

5 Das Impliziertsein beim Betrachten von Bildern, die physische und symbolische Gewalt gegen Menschen zeigen, kann dabei nicht von konkreten Genealogien rassistischer Gewalt und der spezifischen Positionierung sowie den verkörperten Erfahrungen der Betrachterin losgelöst werden. Bilder der Gewalt, die an Schwarzen Körpern verübt wird, sind als öffentliche Spektakel und als Objekte des weißen Blicks elementarer Bestandteil rassistischen Terrors in Post-/Versklavungsgesellschaften (Hartman 1997). Elizabeth Alexanders (1994) Frage „Can you be black and look at this?" verweist auf die 
wortung identifizierte Haltung selbstreflexiv und forschungsethisch in eine politische Theorie der Migration und der Grenze unter den Bedingungen globaler postkolonialer Ungleichheiten übersetzen ließe, erfordert eine theoretische Debatte, die noch weitestgehend aussteht. In diesem Beitrag artikuliert sich die theoretische Verantwortung darin, Migration und Flucht nicht als Krise der Demokratie zu problematisieren, sondern erzwungene Immobilität und die „Unmöglichkeit, einen Ort zu verlassen“ als existentielle Bedrohung für Menschen zu verstehen (Bhabha 2019, 32). Gegenstand der Kritik ist die Politik der Verletzung bis hin zum wissentlichen und straflosen Sterbenlassen von Menschen. ${ }^{6}$

Damit ist auch ein großen Teilen der politischen Theorie gegenläufiges, erweitertes Verständnis von Migration als grenzüberschreitender Mobilität verbunden, das sich einer begrifflichen Unterscheidung in vermeintlich freiwillige Migration einerseits und erzwungene Flucht andererseits entzieht. Diese erweist sich nicht nur als empirisch unhaltbar, wie kritische Migrationsforscher*innen entlang der Heterogenität und Komplexität von Ursachen und Intentionen, Prozessen und Praktiken grenzüberschreitender Bewegungen betonen (Hess et al. 2017, 6). Die scheinbar objektive, da juridische Begriffsbestimmung wird darüber hinaus mobilisiert, um politische und moralische Grenzziehungen und Ausschlüsse zu legitimieren (Crawley und Skleparis 2018, 59) und menschenrechtliche Verpflichtungen der EU-Staaten gegenüber geflüchteten Menschen zu negieren (Benhabib und Sierakowski 2015). Sie wird eingesetzt, um spezifisch situierte grenzüberschreitende Bewegungen zu kriminalisieren und die Entrechtung und strafrechtliche Behandlung bestimmter grenzüberschreitender Menschen als ge-

körperlichen und kulturellen Traumata, die sich in Schwarze Körper und Kollektive eingeschrieben haben und im Moment des Betrachtens, in affektiver Verbundenheit und im Wissen um die eigene potentielle Verletzlichkeit durch ebendiese Akte der Gewalt, aktualisiert werden.

6 Die Frage, welchen Gegenstand die Theoretikerin der Kritik unterzieht und welche ethische Beziehung sie zum Forschungsgegenstand praktiziert, muss dabei auch vor dem Hintergrund eigener und transgenerational tradierter traumatischer Grenzerfahrungen situiert werden. Die theoretischen Überlegungen in diesem Beitrag sind auch durch die familienbiographische Erfahrung vermittelt, der genozidalen Vernichtung nur dadurch entkommen zu sein und zu leben, weil einem Rettungsschiff mit armenischen Geflüchteten aus dem Osmanischen Reich die Einfahrt in den sicheren Hafen von Port Said am afrikanischen Ufer des Mittelmeers gewährt wurde. 
rechtfertigt erscheinen zu lassen. „Migrant*in“ und „Flüchtling“ sind daher als Kategorien einer „Politik des Sortierens und Regierens“ (Hess et al. 2017, 6) zu verstehen, die nicht losgelöst von rassistischen Diskursen betrachtet werden können. Eine politische Theorie der Migration und Grenze, gerade in ihrer normativen Ausrichtung, müsste daher zunächst die oft unhinterfragte Verwendung dieser Kategorien klären sowie ihr disziplinäres Selbstverständnis im Verhältnis zu und möglicherweise in Komplizenschaft mit gewaltförmigen Grenzregimen theoretisieren.

Von diesen Prämissen ausgehend leiten folgende Fragen diesen Beitrag: Wie kann die gegenwärtige Katastrophe im Mittelmeer in einer Weise theoretisiert werden, die die koloniale Vergangenheit nicht als „historische Ungerechtigkeit“, als vergangene „moral wrongs“ (vgl. Ivison 2002, 95-111) rückdatiert und moralisiert, sondern entlang ihrer Dis-/Kontinuitäten in ihrer fortdauernden Präsenz begreift und angemessene politische und ethische Antworten denkbar werden lässt? Wo kann eine Kritik einsetzen, die die Kolonialität der Grenze durchbricht und politische Horizonte jenseits moralischer Appelle sowie humanitärer und juridischer Antworten auf das tägliche Sterben aufscheinen lässt? Und wie lassen sich Demokratie, Staatsbürger*innenschaft und Zugehörigkeit jenseits einer kolonialen und damit immer auch rassistischen Logik denken und praktizieren? Auch wenn auf diese Fragen nur vorläufige Antworten skizziert werden können, argumentiere ich, dass in der Zusammenführung von politischer Theorie mit postkolonialen und Schwarzen Traditionen kritischer Theorie ein begriffliches Register entsteht, das anstelle des Topos der „Flüchtlingskrise“ ein angemesseneres Verständnis der politischen Gegenwart rund um das Mittelmeer ermöglicht und zugleich den Denkraum politischer Theorie erweitert. Die Argumentation wird in drei Schritten entfaltet: Erstens werden Kolonialität und Rasse als zentrale analytische Kategorien postkolonialer und Schwarzer politischer Theorie eingeführt, die die epistemologischen Grenzen eines Großteils politischer Theorie aufzeigen und überschreiten, indem sie ein angemesseneres Verständnis von Grenzen in der postkolonialen Kondition ermöglichen. ${ }^{7}$

$7 \quad$ Anstelle des englischen Begriffs race, der schon lange als sozialwissenschaftliche Kategorie zur Kritik rassifizierter Gesellschafts-, Macht- und Diskursformationen etabliert ist, verwende ich in diesem Beitrag den im deutschen Sprachgebrauch tabuisierten Begriff Rasse. Die Kursivierung markiert, dass der Begriff hier nicht als biologistische Tatsache, sondern ausschließlich als „kritische Wissens- und Analysekategorie“ verwendet wird (Arndt 2015, 187), die historische und gegenwärtige Prozesse der Rassifizierung sicht- und sag- 
Zweitens wird die aus der radikalen Schwarzen Tradition (Robinson 2000) stammende und jüngst im Kontext gegenwärtiger Migrationsrouten von Afrika nach Europa aktualisierte Figur des „Schwarzen Mittelmeers“ (Maio 2014; Smythe 2018; Thompson 2018) als ein Gegenkonzept zum auch in der politischen Theorie allgegenwärtigen Krisennarrativ - sei es als Krise der Demokratie, als Migrationskrise oder als „Flüchtlingskrise“ - aufgegriffen und rekonstruiert. Als eine genuin kolonialismus- und rassismuskritische Epistemologie macht das Schwarze Mittelmeer das aktuelle Geschehen im Mittelmeer in drei verschiedenen, aber miteinander verbundenen Dimensionen lesbar: als eine rassistische Politik der Vergrenzung der Demokratie, als eine ambivalente Politik der Solidarität sowie als eine widerständige Genealogie politischer Imaginationen und Kämpfe um Demokratie und Zugehörigkeit. Zuletzt werden daraus Schlüsse für eine selbstreflexive Auseinandersetzung mit Kolonialität und Rassismus in der politischen Theorie gezogen.

\section{Kolonialität, Rassismus und die Grenzen der politischen Theorie}

Eine postkoloniale Perspektivierung von Migration, Flucht und Grenzen bedeutet nicht nur, ihre Ursachen und Ursprünge vor dem Hintergrund kolonialer Kontinuitäten, gescheiterter Dekolonisierungsprozesse und unlebbarer Lebensbedingungen in den Postkolonien zu betrachten. Sie richtet den Blick auch auf Europa und die postkolonialen Siedlerstaaten. Jacqueline Bhabha bezeichnet den europäischen Kolonialismus als eine der vier großen Triebkräfte menschlicher Migration, der bis in die Gegenwart reichende Verbindungen zwischen den ehemals kolonisierenden und kolonisierten Bevölkerungen geschaffen hat, die über gegenwärtige Migrationsbewegungen, insbesondere koloniale und rassifizierte Arbeitsmigrationsregime, sichtbar werden (Bhabha

bar macht. Ich folge damit der Überlegung Nicola Lauré Al-Samarais und Fatima El-Tayebs, dass ein Ausweichen auf den englischen Begriff Rassismus in deutschsprachigen Kontexten entnennt und einer adäquaten Analyse entzieht (Lauré al-Samarai 2005, 133). Auch euphemistische Ersatzbegriffe wie „Ethnizität“ und „Migrationshintergrund“ haben den Effekt, das europäische „Schweigen über Rasse“ (vgl. Lentin 2008) und das hartnäckige politische wie theoretische Verleugnen von Rassismus in Europa zu verstärken. Gleichwohl steht eine notwendige Verständigung über die Verwendung dieses Begriffes in den deutschsprachigen Sozial- und Geisteswissenschaften, auch vor dem Hintergrund der genozidalen Verflechtungen von Rassismus und Antisemitismus in der deutschen Geschichte, noch aus. 
2019, 20). Ina Kerner weist zudem auf die bis heute anhaltenden, verheerenden Folgen für indigene, kolonisierte und versklavte Bevölkerungen hin, die der europäische Siedlungskolonialismus als historisch beispiellose Massenmigration hinterlassen hat (Kerner 2019, 203). Diese in der politischen Theorie vernachlässigten Kontinuitäten und Verbindungslinien zu zentrieren, ermöglicht es, die kolonialen Dimensionen gegenwärtiger Grenzregime zu erfassen, die sich nicht nur entlang nationalstaatlicher Grenzen manifestieren, sondern weit ins Innere Europas hineinwirken. Zentrale Erkenntniskategorien postkolonialer Kritik sind dabei Kolonialität und Rasse. Dass diese bislang kaum Eingang in das Begriffsrepertoire politischer Theorie gefunden haben, hat, gerade im Forschungsfeld Migration und Grenzen, erhebliche analytische wie normative Auslassungen, Verkürzungen und Verzerrungen zur Folge. Dies gilt besonders für den weit verbreiteten Strang der normativen politischen Theorie in einer anglophonen liberalen Tradition, der Migration und Flucht unter normativen Gesichtspunkten diskutiert, um zu einer moralisch gerechtfertigten Einwanderungs- und Grenzkontrollpolitik zu gelangen (siehe etwa die Beiträge in Fine und Ypi 2016). Dass das konzeptionelle Ausblenden von Kolonialität und Rasse sowohl eine angemessene Analyse und Kritik von Migrations- und Grenzregimen verhindert als auch zu fragwürdigen normativen Politikentwürfen führt, lässt sich exemplarisch anhand einer prominenten Position zur aktuellen „Flüchtlingskrise“ verdeutlichen.

In seinem Buch Strangers in Our Midst: The Political Philosophy of Immigration fragt David Miller (2016) aus der privilegierten Perspektive der Aufnahmegesellschaft, wie „wir, Bürger*innen der reichen Nationen“, auf den Anspruch von Millionen von „Fremden“ antworten sollen, die in „unsere Gesellschaften“ einwandern wollen. Millers politische Theorie der Migration operiert dabei mit mehreren theoretisch unausgewiesenen Prämissen: mit einer sedentären Ontologie (vgl. Sheller 2018, 20), einer etatistischen Epistemologie und einem methodologischen Nationalismus (vgl. Sager 2014). Sesshaftigkeit wird als Grundlage von Staatsbürger*innenschaft normalisiert und die nationalstaatlich verfasste Gesellschaft als unhinterfragte Einheit sozialer Organisation vorausgesetzt. Damit wird Migration als eine Anomalie oder als ein moralisches und politisches Problem behandelt, das es „analytisch zu fassen und politisch zu regulieren, zu kontrollieren und im Zweifelsfall zu begrenzen gilt“ (Kerner 2019, 202). Indem Miller Staatsbürger*innenschaft als ein proprietäres Verhältnis zwischen Nationalstaat und der sesshaften Bevölkerung konzipiert, formuliert er auf der Grundlage eines liberalen Nationalismus und eines schwachen moralischen Kosmopo- 
litismus einen Vorschlag für eine Migrationspolitik, die auf dem Vorrang nationalstaatlicher Gerechtigkeit gründet und die Privilegien der Bürger*innen durch ein strenges Grenzregime schützen soll. Um diese Position als gerechtfertigt erscheinen zu lassen, ist es jedoch notwendig, die durch koloniale Gewalt entstandene globale Verteilung von Reichtum und Armut als einen „geographischen Zufall“ (Miller 2016, 173) zu bagatellisieren und sie mit der zunehmend umstrittenen moralischen Unterscheidung zwischen politisch Verfolgten und ökonomischen Migrant*innen zu untermauern (kritisch dazu Kukathas 2016, 255; Gutiérrez Rodríguez 2018, 19).

Tatsächlich haben gegenwärtige Versuche, nichteuropäische Menschen aus dem Geltungsbereich der Genfer Flüchtlingskonvention auszuschließen, indem ihnen der Status eines politischen Flüchtlings abgesprochen wird, selbst eine koloniale Entstehungsgeschichte, wie Lucy Mayblin rekonstruiert. Sie spiegeln die Bestrebungen der europäischen Kolonialmächte wider, die Konvention mit einer „Kolonialklausel“ zu versehen, um gezielt sowohl nichteuropäische Menschen aus der Definition eines Flüchtlings als auch europäische Menschheitsverbrechen in den Kolonien aus dem Geltungsbereich der Konvention auszunehmen (Mayblin 2014, 428). Diese Logik wird unhinterfragt von Miller übernommen. Basierend auf der Unterscheidung zwischen politisch Verfolgten und ökonomischen Migrant*innen wird das Ertrinken im Mittelmeer als eine „menschliche Tragödie“ entpolitisiert (Miller 2016, 57). Die zunächst befürwortete Rettung aus humanitären Gründen wird im selben Atemzug durch den bereits hinreichend widerlegten populistischen Mythos (vgl. Steinhilper und Gruijters 2017) konterkartiert, die Seenotrettung schaffe erst einen Anreiz für die gefährliche Reise nach Europa (Miller 2016, 171). Millers Entwurf einer normativ begründeten Grenzkontrollpolitik kommt damit der Rettungsbootethik Hardins sehr nahe, ja sie wird implizit zu einer Ethik des „left-to-die boat“.

Nun könnte man einwenden, dass andere Theorieansätze, insbesondere genealogische, historisch-materialistische und postfundamentalistische, diesen theoretisch ebenso wie politisch und moralisch fragwürdigen Prämissen und Schlüssen entgehen. „Vor allem mögen wir es nicht, wenn man uns ,Flüchtlinge“ nennt“, so beginnt Hannah Arendts Nachdenken über die conditio humana ausgehend von ihrer eigenen Fluchterfahrung als verfolgter Jüdin (Arendt 2018, 9). Die von ihr beschriebene grundlegende Spannung zwischen Menschenrechten und Staatsbürger*innenrechten wirkt bis heute als ein zentrales politisches Phänomen fort, wie sich besonders drastisch im Fall des „left-to-die boat“ gezeigt hat. Arendts Überlegungen zum „Recht, Rech- 
te zu haben“ (Arendt 2017) werden daher im Lichte der Rechtsentwicklung seit dem Ende des Zweiten Weltkriegs, insbesondere der Kodifizierung der Menschenrechte und der Genfer Flüchtlingskonvention, und der massenhaften Illegalisierung von Migrant*innen weitergedacht (Benhabib 2008, 2016; Gündoğdu 2015; Menke 2016; Schulze Wessel 2017). Vor dem Hintergrund der Einsperrung von Migrant*innen in Lager und der tödlichen Dimension von Grenzregimen werden auch Michel Foucaults Analytik der Biopolitik und der Gouvernementalität (Sanyal 2017; Mavelli 2017) sowie Giorgio Agambens Konzepte des nackten Lebens und des Lagers aktualisiert (Schulze Wessel 2017; Oberprantacher 2016a). Doch auch in der Tradition von Arendt, Foucault und Agamben stehende Theoretisierungen von Migration reproduzieren zumeist die Auslassung von Kolonialität und Rasse als analytischen Kategorien, die die kritische europäische Theoriebildung seit den 1960er Jahren durchzieht und von Alexander Weheliye als Ausdruck einer theoretischen Provinzialität und begrifflichen Armut kritisiert wird (Weheliye 2014, 63).

Insbesondere die Position von Schwarzsein und damit verbundene existentielle Fragen Schwarzen (Über-)Lebens sind in der europäischen politischen Theorie in ihrer Abwesenheit präsent, sowohl als Gegenstand der Theoriebildung als auch in Form von Schwarzen Theoretiker*innen, die entweder nicht rezipiert oder nicht zitiert und damit unsichtbar gemacht werden. Wie Joy James argumentiert, sind gerade Arendts und Foucaults Theorien durch einen „weißen Solipsismus“ getrübt (James 2016, 266), da sie weder die gelebte Erfahrung und Theoretisierung von Schwarzsein in ihren begrifflichen Apparat eingeführt noch die intellektuelle Auseinandersetzung mit Schwarzen Zeitgenoss*innen gesucht haben. Weheliye konstatiert zudem, dass Foucaults und Agambens Werk zum Standardrepertoire der Auseinandersetzung mit Rassismus, Exklusion, genozidaler Gewalt und Lagern gehört, während Theorietraditionen und -korpora von Schwarzen Menschen und People of Color zu genau diesen Fragen systematisch ignoriert werden. Diesen Umstand, der als epistemische Ignoranz, wenn nicht sogar als epistemische Gewalt bezeichnet werden kann, führt Weheliye darauf zurück, dass Foucault und Agamben gerade deshalb theoretische Glaubwürdigkeit zugesprochen wird, weil sie von einer vermeintlich rassistisch unmarkierten, universellen Position sprechen und Rasse als kritische Denkkategorie ausschließen (Weheliye 2014, 6-8). ${ }^{8}$

8 Für eine ausführlichere Problematisierung der Abwesenheit postkolonialer Kritik in Agambens Theoretisierung des „Flüchtlings“ als politischer Grenzfi- 
Die Erklärungskraft der ohne ein theoretisches Verständnis von Kolonialität und Rasse operierenden Ansätze verfehlt jedoch kategorial deren konstitutive Bedeutung für die politische Verfasstheit der Gegenwart, weshalb sie auch hinsichtlich der „Kolonialität der Migration“ (Gutiérrez Rodríguez 2018) und der rassifizierten sowie rassifizierenden Dimensionen von Grenzregimen (vgl. Browne 2015) an ihre Grenzen stoßen. Der Begriff der Kolonialität erlaubt es, auch nach dem formalen Ende des Kolonialismus fortwirkende Formen kolonialer Macht zu benennen. Nach Aníbal Quijano, der den Begriff prägte, bezeichnet Kolonialität ein genuin modernes „Muste[r] von Machtbeziehungen“ (Quijano 2019, 25), das auf der rassistischen Kodierung und Klassifizierung der Differenzen zwischen kolonialen Eroberern und Eroberten beruht. Diese wurde der kapitalistischen Kontrolle von Arbeit zugrunde gelegt, um die globale Hegemonie Europas zu sichern. Als eine Form der Macht, die aufs Engste mit Kapitalismus und Eurozentrismus verbunden ist, wirkt Kolonialität sich bis heute im Alltagsleben der gesamten Weltbevölkerung aus. Dass diese Form der Macht in der politischen Theorie nicht berücksichtigt wird, führt zu folgenreichen Auslassungen, wie Siba N. Grovogui in seiner Kritik am „westphalian commonsense“ (Grovogui 2002, 316) verdeutlicht. Indem der westfälische Commonsense die Entstehung einer modernen internationalen Staatenordnung auf die Geographie eines kontinentalen Europa begrenzt, unterschlägt er den ontologischen Vorrang von kolonialer Eroberung und imperialistischer Beherrschung für die Herausbildung moderner Staatlichkeit, Souveränität und Subjektivität (so auch Pasha 2011, 220-221). Die „Fiktion“ des westfälischen Staatensystems (Achiume 2019, 32) verunmöglicht es damit, die kolonialen Kontinuitäten und die rassistischen Implikationen gegenwärtiger Migrations- und Grenzregime zu erfassen. Im Kontext von Migration wird Kolonialität als eine Politik der Mobilitätskontrolle unter den Bedingungen globaler Ungleichheiten und damit einhergehender ungleicher Mobilitätsmuster wirksam: zum einen als rassifizierte, kapitalistische Arbeitsmigrationsregime (für den deutschen Kontext siehe Ha 2003), zum anderen als eine gewaltvolle Politik der Immobilisierung (Sheller 2018, 41), die über rassistische und rassifizierende Praktiken des Überwachens und Polizierens von Grenzen durchgesetzt wird (Browne 2015, 16). Die Kolonialität von Grenzen manifestiert sich dabei sowohl in

gur siehe David Farrier und Patricia Tuitt (2013). Für eine rassismuskritische Revision von Foucault im Kontext rassifizierender Mobilitätskontrollen siehe Simone Browne (2015). 
territorialen Staatsgrenzen als auch in der fortwirkenden kategorialen Unterscheidung von Menschen auf Grundlage rassistischer Differenzpostulate (vgl. Kerner 2019, 204), die nunmehr über die vordergründig „farbenblinde“ Differenzierung in Migrant*in und Flüchtling kodiert sind.

Die meisten Denkschulen gegenwärtiger politischer Theorie arbeiten implizit mit einem theoretisch unausgewiesenen Konzept von „Farbenblindheit“ (kritisch dazu Vacano 2012, 142). Dass Rasse eine zentrale Konstruktion einer europäischen Wissens- und Herrschaftsordnung ist, die sich unwiderruflich in das Politische der Moderne eingeschrieben hat, ist dagegen eine der Grundannahmen Schwarzer politischer Theorie. ${ }^{9}$ Rasse wird dabei nicht als objektive Tatsache, sondern als eine politische Kategorie verstanden, die sich als biologische verkleidet (Weheliye 2014, 51). Rasse ist demzufolge keine Eigenschaft phänotypisch voneinander zu unterscheidender Menschen, sondern eine Relation der Herrschaft, eine dynamische Konstellation politischer und sozialer Beziehungen, die das Ergebnis kolonialer Differenzierungen durch anthropologische Abhandlungen, rechtliche Kodifizierungen und administrative Maßnahmen im Gefolge der europäischen Kolonisierung der Welt ist (Hesse 2007, 655). Folgt man Achille Mbembe, bildet Rasse nicht nur den Hintergrund des Nachdenkens über Europas Beziehungen zur außereuropäischen Welt. Rasse ist vielmehr der verleugnete Kern des europäischen politischen Denkens über Liberalismus, Universalismus, subjektive Rechte, nationale Gemeinschaft und staatliche Organisation, auf dem eine bis heute fortwirkende „Welt der Rassen“ errichtet wurde (Mbembe 2014, 110-111).

Diese gleichzeitige Konstruktion und Verleugnung von Rasse in der modernen europäischen politischen Theorie kann mit Barnor Hesse als „epistemologische Rassifizierung“ verstanden werden. Sie wirkt dadurch fort, dass die konstitutive Bedeutung des europäischen Kolonialismus und Rassismus für die Konstruktion Europas konsequent ausgeblendet und Europa weiterhin normativ über Nichteuropa identifiziert und erhoben wird (Hesse 2007, 656). Der eurozentrische Rekurs auf analytische Fiktionen wie den westfälischen Commonsense und auf abstrakte kosmopolitische Ideen ebenso wie die Ontologisierung eines nicht näher bestimmten Lagers und eines rassistisch vermeintlich unmarkierten nackten Lebens bei der gleichzeitigen

9 Schwarze politische Theorie wird hier als deutsche Übersetzung der US-amerikanischen Bezeichnung Black Political Thought eingeführt; für eine Übersicht über das Feld siehe etwa Hanchard (2010). 
theoretischen Vernachlässigung von Rasse verunmöglichen es jedoch, koloniale Gewaltformationen und daraus resultierende extreme politische und ökonomische Asymmetrien zu erfassen, die nicht den Menschen an sich auf die Flucht schicken. Migrant*innen sind immer spezifisch markierte Subjekte innerhalb einer postkolonialen globalen Ordnung rassifizierender Assemblagen (vgl. Weheliye 2014, 72-73), die soziale Beziehungen als ungleiche Machtverhältnisse auf der Grundlage von Rasse in ihrer Intersektion mit anderen Differenzkategorien organisieren und permanent reproduzieren - institutionell, diskursiv, materiell, affektiv, sprachlich, ökonomisch und symbolisch.

Der erweiterte Begriffsrahmen Schwarzer politischer Theorie ermöglicht es, Rassismus und Rassifizierung als genuin politische Phänomene der Moderne - und zwar in ihrer global vorherrschenden Form als weiße Vorherrschaft (Mills 1994) und in ihrer „Banalität“ als institutionalisierte und normalisierte Alltagsphänomene in weiß dominierten Gesellschaften jenseits singulärer Gewaltexzesse (Ferreira da Silva 2017) - der Reflexion zugänglich zu machen. ${ }^{10}$ Gegen den unausgesprochenen Primat von Weißsein als zentraler Macht- und Wissensformation einer kolonialen Moderne, der sich auf einer theoretischen Ebene in der Privilegierung und gleichzeitigen unüberprüften Universalisierung der gelebten Erfahrungen, Empfindungen und Perspektiven als weiß rassifizierter Menschen äußert, zentriert Schwarze politische Theorie Schwarzsein als primäre Erscheinungsform der Rassifizierung und als fundamentale Ausnahme- und Grenzkategorie der Moderne (Weheliye 2014, 3). Sie untersucht die konstitutive Bedeutung von anti-Schwarzem Rassismus für moderne Konzeptionen und Konfigurationen des Politischen, die durch „evasive Konzepte“ wie Multikulturalismus oder Diversität unsichtbar gemacht wird (vgl. Hart 2018, 6). Schwarze politische Theorie überschreitet damit die „kognitiven Grenzen“ (vgl. Mezzadra und Neilson 2013, 16) eurozentrischer politischer Theorie und einer „weißen Epistemologie der Ignoranz“ (vgl. Mills 2007; 2017), deren als „farbenblind“ verstandener universeller Anspruch auf der uneingestandenen affektiven Bindung an Weißsein beruht (hooks 1992) und sich in das Phantasma „nord-

10 Die Begriffe Weißsein und weiß werden kursiv gesetzt, um sie in Abgrenzung zu einem epidermalen Verständnis als eine gesellschaftlich konstruierte Norm, Machtformation und soziale Positionierung zu verstehen. Schwarz wird dagegen groß geschrieben, um eine antirassistische Selbstbezeichnung und ein gegen Kolonialität und Rassismus gerichtetes politisches Selbstverständnis zum Ausdruck zu bringen. 
atlantischer Universalien“ (vgl. Trouillot 2002) verstrickt. Wie lässt sich die gegenwärtige Katastrophe des Ertrinkenlassens im Mittelmeer dagegen aus einer explizit postkolonialen, aus einer Schwarzen theoretischen Perspektive verstehen?

\section{Black Mediterranean: Das Mittelmeer in der Kartographie Schwarzer kritischer Theorie}

Die Figur des Schwarzen Mittelmeers ordnet sich in eine Epistemologie und Genealogie Schwarzer Theoriebildung ein (Smythe 2018, 7), mit der die Flüchtigkeit und das Sterben Schwarzer Menschen vor dem Hintergrund fortwirkender kolonialer und rassistischer Macht- und Herrschaftsformationen und der zentralen Bedeutung von anti-Schwarzer Gewalt für die politische Moderne gedeutet wird. Als ein Ensemble von diasporischen Denktraditionen und praktischen Erfahrungen aus den Befreiungskämpfen Schwarzer Menschen, das im 18. Jahrhundert beginnt und seit den 1960er Jahren in den USA als Black Studies institutionalisiert ist, umfasst Schwarze kritische Theorie (Black Critical Thought) sowohl eine substantielle Kritik der politischen Kategorien der westlichen Moderne als auch ein bedeutsames Archiv sozialer, politischer und kultureller Alternativen (Weheliye 2014, 3). Das verbindende Element unterschiedlicher Traditionen Schwarzer Theorie ist kein epidermales Verständnis von „Hautfarbe“, das selbst als Erfindung einer kolonialen Epistemologie theoretisiert werden muss, sondern der Bezug auf die Tropen „Versklavung“ und „Afrika“ als die beiden zentralen Pole eines geteilten Erfahrungshorizonts und eines zugleich heterogenen und pluralen politischen Imaginären (Scott 2013, 4). Innerhalb dieses weit verzweigten und vielstimmigen Theoriekanons bewegt sich die Debatte um das Schwarze Mittelmeer zwischen der Tradition eines Black Marxism und neueren Ansätzen des Afropessimismus. ${ }^{11}$ Während vor allem Paul Gilroys Konzept des Black Atlantic (Gilroy 1993) mit der transatlantischen Versklavung afrikanischer Menschen als Urszene der Moderne verbunden wird, geht das Konzept des Black Mediterranean auf Cedric J. Robinsons 1983 erschienenes Buch Black Marxism. The Making of the Black Radical Tradi-

11 Dabei stehen Black Marxism und Afropessimismus, die jeweils in sich heterogene und widerstreitende Genealogien und Theoriebestände sind, in einem durchaus spannungsreichen Verhältnis zueinander hinsichtlich ihrer Theoretisierung der Erfahrung und Bedeutung von Schwarzsein, der Möglichkeiten widerständiger Praktiken und alternativer Konzeptionen des Politischen. 
tion zurück. Robinson zeichnet darin die sowohl in liberalen als auch in marxistischen Historiographien verleugnete Vorgeschichte der Entstehung eines modernen kapitalistischen Weltsystems nach, die er im Mittelmeerraum der frühen Neuzeit lokalisiert. Demzufolge bildet die dort praktizierte Versklavung europäischer und afrikanischer Menschen vom 13. bis zum Beginn des 15. Jahrhunderts den historischen Auftakt für die spätere, systematisch organisierte transatlantische Versklavung afrikanischer Menschen (Robinson 2000, 16). Dieses frühe, bereits auf Plantagenökonomie und Zuckerproduktion basierende System der Versklavung wurde im 15. und 16. Jahrhundert durch die Kolonial- und Seemächte Portugal und Spanien im Zusammenspiel mit italienischen und englischen Bankiers, Kaufleuten und Händlern sukzessive in den atlantischen Raum verlagert (Robinson 2000, 92). Im Übergang vom 16. zum 17. Jahrhundert wurde schließlich Versklavung mit Schwarzsein und Freiheit mit Weißsein kodiert und ontologisch über die „color line“ fixiert (Hartman 2007, 5). Wie Robinson aus einer Schwarzen marxistischen Perspektive klarmacht, muss daher der Mittelmeerraum als Entstehungsort eines von Beginn an über Rassifizierung operierenden Kapitalismus (racial capitalism) gelten, der über rassistische Konstruktionen von Weißsein und Schwarzsein und die Dehumanisierung und Kommodifizierung afrikanischer Menschen die symbolische wie ökonomische Konstitution eines weißen Europas ermöglichte, das in der Folge durch rassistische Politiken der Grenzziehung und -kontrolle abgesichert wurde (Kelley 2000, xiii).

In der gegenwärtig als „Flüchtlingskrise“ gedeuteten Konstellation gewinnt das Schwarze Mittelmeer als Analyseeinheit neue Relevanz, um die historisch wie gegenwärtig gewaltvollen bis hin zu tödlichen Erfahrungen Schwarzer Menschen zu theoretisieren. Verkörpert diese genuin koloniale und rassistische Gewaltgeschichte des Mittelmeers in weißen Formationen kritischer Theorie eine konzeptionelle „Totwasserzone“ - „a studious absence of analytical engagement with the protocols of antiblack racism and genocide in Western democracies“ (Saucier und Woods 2014, 57) -, wird das gegenwärtige Mittelmeer in den Linien Schwarzer kritischer Theorie ausgehend von der paradigmatischen Position von Schwarzsein in einer europäisch-afrikanischen Verflechtungsgeschichte kontextualisiert. Die auf Sizilien lebende Literaturwissenschaftlerin Alessandra di Maio prägte in Anlehnung an Robinson durch die Zusammenführung der Konzepte Schwarzsein und Mittelmeer den Begriff des „Schwarzen Mittelmeers“, um damit das Mittelmeer rund um Lampedusa als den zentralen Schauplatz afrikanischer Migrationsrouten zu verstehen, die heute so konstitutiv für die globale Zirkulati- 
on von Kapital-, Ressourcen- und Finanzströmen sind wie die historische Versklavung afrikanischer Menschen, in deren Schatten sie stehen (Maio 2014, 28-29). Das Mittelmeer ist dabei nicht auf die Oberfläche des Meeres begrenzt, sondern erstreckt sich bis in die afrikanischen Länder südlich des Sahel, aus denen die Menschen in Richtung Europa aufbrechen. Die von ihnen eingeschlagenen Migrationsrouten sind identisch mit den „Transsahara“-Routen des früheren Versklavungshandels. Diese nicht kontingente Koinzidenz legt es nahe, Versklavung als eine zentrale und bis heute fortwirkende, aber in einem Großteil europäischer soziologischer und politischer Theorie vernachlässigte Dimension der Macht und als zentrales Paradigma menschlicher Objektivierung zu fokussieren, um Lampedusa und die Polizierung des Mittelmeers zu verstehen, so Khalil Saucier und Tryon Woods $(2014,61)$.

Die fortwirkende Bedeutung von Versklavung für die Gegenwart zu konzeptualisieren ist das Leitmotiv von Theoretiker*innen um Saidiya Hartman und Frank B. Wilderson III, die dem Afropessimismus zugerechnet werden. Ihr zentraler theoretischer Bezugspunkt ist Schwarzsein, das ausgehend von der Ontologie des europäischen Humanismus als strukturelle Position des Versklavtseins gebrandmarkt ist und nicht nur ökonomisch, sondern auch symbolisch die Moderne und ihre emanzipatorischen Versprechen erst ermöglicht (McKittrick 2015b, 2; Wilderson 2010, 58-59). ${ }^{12}$ Afropessimistisch gesprochen müssen die Flüchtigkeit (fugitivity) und das Sterben Schwarzer Menschen (black death) als grundlegende Erscheinungsweisen des „Nachlebens der Versklavung“ (afterlive of slavery; Hartman 2016b, 210) gedeutet werden, in welchem der ontologische Status von Schwarzsein nicht als Subjekt oder Objekt, sondern als moralisch wertloses Abjekt unauflöslich mit Akkumulation und Austausch-/Verwertbarkeit (fun-

Afropessimismus ist eine subversive Aneignung des aus der Entwicklungstheorie stammenden Begriffs, in dem eine grundlegende Skepsis über die „Entwicklungsfähigkeit“ des afrikanischen Kontinents zum Ausdruck kommt (Hart 2018, 19). Obwohl Afropessimismus keine geschlossene Denkschule ist und etwa von Hartman nicht als Selbstbezeichnung gewählt wird, sind die zentralen theoretischen Bezugspunkte Frantz Fanons Phänomenologie des Schwarzseins, Orlando Pattersons Konzept von Versklavung als sozialer Tod, Hortense Spillers' Unterscheidung zwischen Körper und Fleisch in der Erfahrung der Versklavung sowie Hartmans Begriff des Nachlebens der Versklavung (Hart 2018, 17). Zu einer ausführlichen Einführung in Afropessimismus als kritischem Idiom siehe Sexton (2016). 
gibility) verbunden ist (Wilderson 2010, 55). Mit dem aus der Psychoanalyse stammenden Konzept der Abjektion (Hartman und Wilderson 2003, 198), das hier ontologisch als Abspaltung gewendet wird, geht die afropessimistische Kritik weit über eine materialistische Kritik postkolonialer und rassistischer Macht-, Unterdrückungs- und Ausbeutungsverhältnisse hinaus. Mit dem Fokus auf die ontologische Dimension von Schwarzsein erschöpft sich dieses nicht in einem durch Unfreiheit, Ungleichheit und Ausbeutung gekennzeichneten Subjektstatus innerhalb global vermachteter kapitalistischer Asymmetrien und in der Vorenthaltung von Staatsbürger*innenschaft. Schwarzsein markiert vielmehr die Verunmöglichung eines Subjektstatus, ist die Position des Nichtseins, des Unmöglichseins. Diese Verunmöglichung Schwarzen Seins ist eine Folge von Praktiken der Abjektion in und durch Versklavung - Dehumanisierung, Verdinglichung, gewaltvolle Zurichtung -, die Schwarzsein nicht nur zum Objekt machen, sondern aus der symbolischen Ordnung der Moderne abspalten, das heißt, aus einer aufgeklärten politischen, ökonomischen und moralischen Ordnung historisch wie symbolisch „über Bord werfen“ (vgl. Broeck 2018, 84).

In der gewaltvollen Abjektion von Schwarzsein, die zugleich den Subtext weißer Subjektivierung bildet, nimmt das Schiff - vom Sklavenschiff bis zum „left-to-die boat“ - eine besondere Bedeutung als historischer wie gegenwärtiger Ort anti-Schwarzer Gewalt ein. Die Schlauchboote, die aufs Mittelmeer geschickt werden, sind Berichten von Überlebenden zufolge Schauplätze „unmenschlicher Gewalt“: durch das lebensgefährliche und oft tödlich endende Zusammenpferchen auf und unter Deck, durch Erstechen, Ersticken und Erschießen, Über-Bord-Werfen und sexualisierte Gewalt durch Menschenschmuggler*innen (vgl. Sharpe 2016, 55). Die aktuellen Statistiken der Tode erscheinen als Nachhall der Logbücher von Versklavungsschiffen, in denen die Präsenz der kommodifizierten, zur Frachtware gemachten Menschen über die numerische Erfassung der Toten und der Sterbenden hergestellt wird, eine Spur ihres Lebens ausschließlich im Augenblick der Gewalt und des Todes sichtbar wird: „the list, the breathless numbers, the absolutely economic, the mathematics of the unliving" (McKittrick 2015a, 17). Dass die Berichte und Bilder von sinkenden Schiffen und ertrinkenden Menschen inzwischen täglich in den Medien auftauchen, ohne dass daraus politische Konsequenzen gezogen werden, als Minimalforderung die Wiedereinführung der Seenotrettung, scheint die Bedeutung von Schwarzsein als normativ wertlosem Abjekt im politischen Imaginären Europas und die Normalität anti-Schwarzer Gewalt zu bestätigen. Der massenhaft produzier- 
te vorzeitige Tod Schwarzer Menschen in den libyschen Lagern, auf den Booten und im Meer bleibt politisch folgen- und rechtlich straflos.

Diese verschiedenen Erscheinungsweisen anti-Schwarzer Gewalt innerhalb des europäischen Grenzregimes, die afrikanische Migrant*innen in der Verschränkung von Gefangenschaft und Flüchtigkeit von den unterschiedlichsten sozialen Gruppen und politischen Institutionen erfahren, können mit Mbembes Konzept der Nekropolitik verstanden werden. In Abgrenzung zu Foucaults Begriff der Biopolitik erfasst Nekropolitik die Unterwerfungsoperationen souveräner Macht, die menschliche Körper und ganze Bevölkerungsgruppen - und zwar primär über die Kategorie Rasse markierte - der Vernichtung aussetzen (Mbembe 2003, 14). Auch wenn Mbembe die Wirkweisen von Nekropolitik historisch am Beispiel der Plantage und der Kolonie erläutert, die sich über die Rassifizierung von Körpern, Arbeits- und Rechtsverhältnissen konstituierten, geht er von ihrer fortdauernden Persistenz in der postkolonialen Gegenwart aus. Wie sich in der tödlichen Politik gegen flüchtende Menschen vor allem aus afrikanischen Ländern zeigt, operiert auch hier Rasse als der Marker, der über moralische wie ökonomische Register definiertes wertvolles und wertloses Leben, rettungswürdiges und zur Disposition stehendes Leben unterscheidet. Genau dieser radikale Perspektivwechsel vom kontrafaktischen Postulat aufklärerischer Universalismen im Angesicht ihrer täglichen Negation hin zur Identifizierung der Abjektion von Schwarzsein als Universalie einer aufgeklärten Moderne lässt die tödliche Logik des „left-to-die boat“ plausibel erscheinen: „From the vantage of the policed, the Mediterranean is a deathscape, a zone of disposability, not one of simple risk“" (Saucier und Woods 2014, 70).

Der afropessimistische Begriffsrahmen kann damit erfassen, was über Ausbeutung und Entfremdung als den zentralen Kategorien einer klassischen, auf politische Ökonomie begrenzten marxistischen Gesellschaftskritik unreflektiert bleibt: die Frage, wie die libidinöse Identifizierung mit tödlicher Gewalt gegen Schwarze Menschen historisch wie gegenwärtig in einer Weise objektiven Eingang in politische, moralische und ökonomische Ordnungen findet (Sexton 2016, 14), dass Schwarzsein nicht nur aus dem politischen Bereich der Zivilgesellschaft und des Staates ausgeschlossen, sondern - wie sich eingangs in der Rekonstruktion des „Left-do-die boat“-Falls zeigte - außerhalb der moralischen Gemeinschaft einer mit dem Recht auf Leben und anderen unveräußerlichen Rechten geborenen, universellen Menschheit positioniert wird. Schwarzsein konstituiert sich damit als liminal category, als Grenze einer humanistischen Seinsordnung, die bis heute in ihrer genozi- 
dalen Gewalt fortwirkt (Wynter 1994, 67). In der postkolonialen Welt sind es die Geflüchteten und Migrant*innen, die Illegalisierten und in Gefängnissen und Lagern Eingesperrten, die diese Grenzfigur verkörpern, wie Sylvia Wynter konstatiert: „That is, as a category defined at the global level by refugee/ economic migrants stranded outside the gates of the rich countries, as the postcolonial variant of Fanon's category of les damnés“ (Wynter 2003, 261). Fanonianisch gedacht sind auch Mbembe zufolge „die neuen ,Verdammten dieser Erde“ jene, denen das Recht auf Rechte verwehrt bleibt“: Sie „sind das Ergebnis einer brutalen Kontrolle und Selektion, deren rassische Grundlagen bestens bekannt sind“ (Mbembe 2014, 323).

Die Gewalt, die zur Etablierung und Aufrechterhaltung dieser rassistischen Grenzziehung als Struktur der Moderne notwendig ist, wird typischerweise in einer Vergangenheit außerhalb Europas lokalisiert, hauptsächlich auf den Versklavungsplantagen Amerikas - „as Europe's externality, the colonial outside“ (Goldberg 2006, 332). Sie wirkt jedoch nicht nur in den amerikanischen Postversklavungs- und Postsiedlergesellschaften, sondern in Europa selbst, wo anti-Schwarzer Rassismus als elementare Dimension einer kolonialen Moderne fest im politischen Imaginären und in den politischen Institutionen sedimentiert ist. So verstanden ist das zur Disposition stehende Leben Schwarzer Menschen im Mittelmeer weder eine flüchtige Episode der Gegenwart noch eine unerklärliche Abweichung von der Selbstverpflichtung der EU auf die Achtung universeller Menschenrechte. Es ist die historisch akkumulierte Gewalt gegen Schwarze Menschen und ihre Positionierung jenseits der Grenzen der Demokratie, des Rechts und der Moral, die als verleugneter Subtext der politischen Moderne in der unterlassenen Hilfeleistung gegenüber Schwarzen Frauen, Männern und Kindern und in der Kriminalisierung ihrer Lebensrettung fortwirkt. Das europäische Grenzregime ist in den Begriffen Schwarzer kritischer Theorie daher als ein Ensemble von Praktiken der Rassifizierung und der Polizierung zu verstehen, die auf die Aufrechterhaltung Europas nicht nur als militärisch abgesicherter Festung, sondern als einer als weiß rassifizierten, „gebleichten“ politischen Einheit abzielen (vgl. Gilroy 2004, xii). 


\section{Das Schwarze Mittelmeer und die rassistische Politik der Vergrenzung}

Das Schwarze Mittelmeer als eine Epistemologie der Grenze ermöglicht es, Migrations- und Grenzregime als postkoloniale Praktiken der Rassifizierung zu verstehen, die in westlich liberalen Demokratien den Ein- und Ausschluss in und aus der politischen Gemeinschaft entlang rassistischer Grenzziehungen regulieren und reproduzieren und als solche den Regel- und nicht den Ausnahmefall bilden. Dass Europa, dessen geographische wie kulturelle Grenzen schon immer uneindeutig und umstritten waren, sich als eine kohärente symbolische und politische Einheit konstituiert hat, muss dabei selbst als Ergebnis von epistemologischen und gouvernementalen Prozessen der Rassifizierung und der ontokolonialen Erfindung Europas und Nichteuropas in der Begegnung mit dem kolonisierten und versklavten Anderen seit dem 16. Jahrhundert verstanden werden (Hesse 2007, 658). David Theo Goldberg bezeichnet diese Prozesse als „racial Europeanization“ und als „Europeanization of Europe“ (Goldberg 2006, 352). Ihr Ergebnis ist, dass Europa und Europäischsein mit den Bedeutungen zivilisiert, überlegen, fortschrittlich belegt und mit den Signifikanten weiß, männlich und christlich überschrieben wurden, woraus folgte, dass das als Antithese konstruierte Nichteuropa als nichtwei $\beta$ kodiert wurde und letztlich alle als nichtwei $\beta$ rassifizierten Menschen in Europa als nichteuropäisch und nichtzugehörig definiert wurden und weiterhin werden (Goldberg 2006, 352; Hesse 2007, 655). Kolonialität als Ontokolonialität (Hesse 2007, 659) ist damit der notwendige Entstehungskontext der gleichzeitigen Rassifizierung und Universalisierung Europas als einer homogenen und klar definierten Einheit, die die geographisch unbestimmten Grenzen Europas entlang der Differenzkategorie Rasse vereindeutigt als auch politische und kulturelle Zugehörigkeit rassistisch begrenzt. Die koloniale Konstitution und Begrenzung Europas erfolgte folglich nicht primär über die militärische Absicherung willkürlich gezogener territorialer Grenzen, sondern vielmehr über die Kategorie Rasse, so Goldberg (2006, 358): „Borders are constituted through race, the biopolitical technology par excellence, fashioning the foreigner, the stranger, the not-belonging“.

Die Polizierung rassifizierter Grenzen in Europa findet sich bereits in historischen Rechtstexten vorgezeichnet, die auf die Reinhaltung Europas als weißer Einheit abzielten: 1777 legte ein königlicher Erlass mit dem Titel „La Police des Noirs“ fest, dass es Schwarzen Menschen ebenso wie Menschen of Color unabhängig von ihrem Rechtsstatus als Freie oder Versklavte verboten 
ist, den Boden der französischen Metropole zu betreten (Peabody 1997). Bei Zuwiderhandlung seien sie sofort zu verhaften und über den nächsten Hafen abzuschieben. Diese institutionalisierte und verrechtlichte Politik der Grenze wirkt, wenn auch anders konfiguriert, im gegenwärtigen Mittelmeer fort. Mit Simone Browne können die Überwachung des Mittelmeers und damit verbundene Technologien sowie politische Maßnahmen als eine diskursive und materielle Praxis sozialer Kontrolle verstanden werden, deren Ziel es ist, Grenzen und Körper entlang rassifizierter Linien in ihrer Intersektion mit Klasse, Geschlecht, Sexualität und anderen Differenzkategorien zu reifizieren. Mit dem Konzept „racializing surveillance“ macht Browne deutlich, dass das Grenzregime nicht nur rassistisch ist, sondern permanent rassifizierte Andere herstellt (Browne 2015, 42). Das Überwachen und Polizieren von Schwarzsein stellt darin keine Ausnahmeerscheinung dar, sondern die soziale und politische Norm, die reguliert, welche Körper an welchem Ort anwesend sein dürfen (Browne 2015, 16). Mit der Aufhebung der nationalstaatlichen Grenzen zugunsten eines gemeinsamen Schengenraums, die zu einer verstärkten Überwachung und Sicherung der EU-Außengrenzen geführt hat, wurde die Grenzkontrolle zudem in Form von Racial Profiling ins Innere der EU verlagert (El-Tayeb 2008, 651). Diese informelle Praxis des Polizierens auf der Grundlage pseudobiologischer und implizit rassifizierter Konzeptionen von EU-Staatsbürger*innenschaft in den europäischen Traditionslinien unterschiedlicher, aber miteinander verbundener Rassismen produziert klar identifizierbare Außenseiter*innen und bedrohliche Andere. Diejenigen, die als „Others-From-Without“ imaginiert und als von außen kommend repräsentiert werden, sind dabei immer auch „Others-From-Within“ (El-Tayeb 2008, 658-659): jüdische und Schwarze Menschen, Muslim*innen bzw. muslimisch gelesene Menschen und Rom*nja und Sinti*zze, die alle schon immer zu Europas Geschichte und Gegenwart gehört haben. Bereits die vermeintlich harmlose Nachfrage „Wo kommst du her?“ als alltägliche Variante von Racial Profiling und die Kategorie „Migrationshintergrund“ für rassifizierte Europäer*innen, die selbst nie migriert sind, verweisen laut El-Tayeb darauf, dass das vorherrschende Verständnis von Europäischsein nicht ohne das rassifizierte Andere als Außen existieren kann (El-Tayeb und Vanessa E. Thompson 2019, 322). Schwarzsein wird in dieser Logik zur Repräsentation von Nichtzugehörigkeit, von Nichtstaatsbürger*innenschaft, zur nicht inkorporierbaren Alterität einer weißen Demokratie. Innerhalb des EU-Raums fungiert Rasse damit nicht nur als externe, sondern auch als interne Grenze der Demokratie. 
Eine historisch-materialistische Analyse des europäischen Grenzregimes ist aus dieser Perspektive nicht ausreichend, um zu erfassen, dass das Polizieren der Festung Europa eine soziale Methodologie ist, die auf anti-Schwarzer Gewalt beruht (Saucier und Woods 2014, 75). Die Mobilisierung eines fest sedimentierten rassistischen Imaginären trifft dabei auf neue Formen rassistischer Gouvernementalität durch Migrationskontrolle, die die Anderen der Nation/Europas/des Westens immer wieder neu in rassifizierenden Begriffen konfiguriert (Gutiérrez Rodríguez 2018, 24). Dadurch wird, so Gloria Wekker (2009, 283-284), der Mythos eines „reinrassigen“, nämlich homogen weißen Europas aufrechterhalten, während gleichzeitig die statistisch nachgewiesene Präsenz von etwa 18 Millionen Schwarzen Menschen in Europa aus dem hegemonialen europäischen Selbstverständnis ausgeschlossen und innerhalb der rassifizierten Grenzen Europas undenkbar wird. Diese „numerische Auslöschung“ (vgl. Linke 2011, 126) kann als ein Echo der dehumanisierenden Vergangenheit der Versklavung gedeutet werden, die in Europa noch immer weitgehend uneingestanden ist. Mit Mbembe kann sie aber auch als Teil einer grundlegenderen Selbsttäuschung der liberalen Demokratie verstanden werden: „Ausschluss, Diskriminierung und Selektion im Namen der Rasse sind [...] weiterhin strukturierende wenn auch vielfach geleugnete - Faktoren der Ungleichheit, der Verwehrung von Rechten und der aktuellen Herrschaft, und das selbst in unseren Demokratien. Man kann auch nicht so tun, als hätte es Sklaverei und Kolonisierung nie gegeben oder als wäre das Erbe dieser finsteren Zeit vollkommen beseitigt. Die Umwandlung Europas in eine Festung etwa und die gegen Ausländer gerichteten Gesetze, die auf dem alten Kontinent zu Beginn dieses Jahrhunderts verabschiedet wurden, haben ihre Wurzeln in einer Ideologie der Selektion zwischen verschiedenen Menschenarten, die man allerdings eher schlecht als gut zu maskieren versucht“ (Mbembe 2014, 322).

Europa als begriffliche Einheit von Staatsbürger*innenschaft, Säkularismus, Demokratie und einem affektiv besetzten und zugleich geleugneten Weißsein (Hesse 2009, 292) kann vor diesem Hintergrund als Ergebnis von tiefliegenden Praktiken der Rassifizierung verstanden werden, die nicht im Widerspruch zur Demokratie in den EU-Mitgliedsstaaten wirkt, sondern konstitutiv für die Entstehung und Begrenzung der europäischen Demokratie war und ist. Aus dieser Sicht sind, so Mbembe (2018) in deutlichem Kontrast zu einem Großteil normativer politischer Theorien, „nicht die Migranten das Problem, auch nicht die Flüchtlinge oder die Asylsuchenden. Sondern Grenzen. Alles beginnt mit ihnen, und alle Pfade führen zu ihnen zurück. Sie 
sind nicht mehr nur eine Markierungslinie, die souveräne Gebiete trennt. Sie sind zunehmend die Bezeichnung für die organisierte Gewalt, die dem heutigen Kapitalismus und unserer Weltordnung zugrunde liegt - durch die Frauen, Männer und Kinder zu Ausgestoßenen werden.“ Diese Politik der „Vergrenzung“, wie Mbembe sie bezeichnet, macht bestimmte Räume für bestimmte Menschengruppen zu undurchquerbaren und zu unlebbaren Orten. Sie sichern „Europa allein das Privileg der globalen Besitznahme und ungehinderten Bewegungsfreiheit“ (ebd.). Mbembe macht damit explizit, was in der europäischen politischen Theorie kaum theoretisiert wird: dass die Politik der Vergrenzung auf der Relationalität von erzwungener Immobilität auf der einen, rechtlich abgesicherter Bewegungsfreiheit auf der anderen Seite beruht und in den kolonialen Kontinuitäten eines grenzüberschreitenden rassifizierenden Kapitalismus steht.

\section{Das Schwarze Mittelmeer und die Grenzen der Solidarität}

Angelehnt an Blumenbergs Studie Schiffbruch mit Zuschauer (1979) über die Bedeutung des Schiffbruchs als Metapher in der Geschichte des europäischen Denkens greift Paul Gilroy die Bedeutung der eingangs aufgeworfenen Erfahrung der Zeug*innenschaft angesichts der fortgesetzten Katastrophe des Ertrinkens im Mittelmeer auf (Gilroy 2018, 16-17). Gilroy situiert seine Überlegungen im Kontext der tödlichen Performativität einer neofaschistischen und rassistischen populistischen Rhetorik in einem postkolonialen Europa, die geflüchteten Menschen symbolisch ihre Humanität abspricht, um ihnen in letzter Konsequenz die Seenotrettung und damit das Recht auf Leben zu verweigern. Wie könnte, so fragt Gilroy, die Rettung ertrinkender Menschen im Geiste einer - in den Worten Sylvia Wynters - „Wiederverzauberung des Humanismus“ imaginiert und praktiziert werden? Dazu führt er eine beispielhafte „Banalität des Guten“ an (ebd., 16). Ein Soldat, der außerhalb seiner Dienstzeit Zeuge eines Schiffbruchs vor der griechischen Küste wurde, rettete im April 2015 - instinktiv, ohne nachzudenken, wie er sagte - zwanzig von insgesamt dreiundneunzig geflüchteten Menschen aus Syrien und Eritrea vor dem Ertrinken. Gilroy warnt davor, dieses Ereignis als eine Politik des Mitleids misszuverstehen, wie sie durch die gewohnten Mechanismen der medialen Inszenierung evoziert wird. In der Berichterstattung wurde der Vorfall individualisiert und emotionalisiert, indem die Rettung einer namentlich genannten schwangeren Schwarzen Frau fokussiert und Bilder des Leidens nichteuropäischer Menschen produziert wurden, ohne 
dieses Leiden in die geopolitischen Verschränkungen zwischen Europa, Afrika und dem Nahen Osten einzuordnen. Im Gegensatz zu einer dadurch vermittelten Politik des Mitleids eröffne die menschliche Begegnung inmitten der Gewalt des Meeres, die gemeinsame Erfahrung, keinen Boden unter den Füßen zu haben, so Gilroy, den Horizont eines wenn auch fragilen, so doch alternativen Humanismus, der auf einer geteilten Verletzlichkeit und einer ursprünglichen Verantwortung für die Andere gründet: „We are afforded a glimpse of a vulnerable, offshore humanity that might, in turn, yield an offshore humanism." (ebd., 18)

Doch sind der mit einer EU-Bürgerschaft und folglich mit einklagbaren rechtlichen und moralischen Ansprüchen ausgestattete Soldat und die schwangere eritreische Frau, die unter Lebensgefahr für sich und ihr ungeborenes Kind im Schlauchboot das Mittelmeer durchquert, tatsächlich in der gleichen Weise verletzliche Mitglieder einer universellen Menschheit? In scharfem Kontrast zu Gilroys Suche nach einem antirassistischen Humanismus ist aus einer afropessimistischen Perspektive eine unvermittelte menschliche Begegnung auf dem Meer nicht denkbar. Unabhängig von einem intuitiven individuellen moralischen Gestus kann das Zusammentreffen auf hoher See nicht jenseits der Gewalt eines Grenzregimes gedacht werden, das als Manifestierung eines anti-Schwarzen Rassismus auf der strukturellen Verunmöglichung einer ethischen Haltung gegenüber Schwarzen Menschen beruht (vgl. Wilderson 2010, 131). Die mediterrane Nekropolitik, die auf der Rassifizierung von Menschen und der Normalisierung ihres Sterbens beruht, ist demzufolge nicht durch einen moralisch motivierten Humanismus weiß positionierter Verbündeter zu überwinden. „The rescuers' sympathy does not mitigate Fortress Europe's death-dealing policies", so kommentiert Christina Sharpe Zeitungsberichte über einen Schiffbruch ohne positiven Ausgang. Die Rettungstaucher*innen waren starr vor Schreck, als sie den leblosen Körper einer Schwarzen Frau vom Meeresboden bargen. Sie hatte im Moment des Ertrinkens ihr Kind geboren (Sharpe 2016, 57). ${ }^{13}$ Sharpe zu-

13 Die häufige mediale Repräsentation von Schwangerschaft und Geburt auf der Flucht, die die gewohnte Repräsentation des „Flüchtlings“ als männlich aufbricht, unterstreicht die Notwendigkeit, Grenzregime als miteinander verschränkt rassifizierte und vergeschlechtlichte Gewaltformationen zu begreifen, in denen koloniale Geschlechter- und Bevölkerungspolitiken fortwirken. So verweisen die Diskurse um „Anchor Babies“, um unverantwortliche Schwarze Mutterschaft oder die Annahme, dass Schwangerschaft auf der Flucht primär eine Folge sexualisierter Gewalt sei (kritisch dazu Barbara et 
folge sind diese medial transportierten Katastrophenbilder Schwarzen Leidens und Sterbens rund um Lampedusa gerade nicht der visuelle Ausdruck eines authentischen moralischen Empfindens. Sie folgen vielmehr einer historisch gewachsenen Dramaturgie und Ästhetik rassifizierter Imaginationen, die Bilder Schwarzen Leidens als Objekte öffentlichen Konsums für ein weiß positioniertes Publikum produzieren. Damit bilden sie, ähnlich der weißen abolitionistischen Bewegung zur Abschaffung der Versklavung, eine Hinterbühne für Inszenierungen humanitärer Rettungsaktionen, in denen die symbolischen Grenzen zwischen dem weißen Subjekt der Menschenrechte und dem Schwarzen Objekt von humanitärer Hilfe und Wohltätigkeit, wenn nicht gar dem sterbenden Schwarzen Abjekt reproduziert werden (Sharpe 2016, 53).

„Der Flüchtling“ ist im Kontext des Schwarzen Mittelmeers insofern kein Sinnbild einer abstrakt imaginierten Menschheit. Im Diskurs um die „Flüchtlingskrise“, und zwar sowohl in affirmativen wie in kritischen und solidarischen Bezugnahmen, wird „der Flüchtling“ zur rhetorischen und ästhetischen Trope, die das Andere Europas repräsentiert und gerade dadurch europäisches Weißsein als Norm reproduziert (Gutiérrez Rodríguez 2018, 24). Besonders deutlich wird diese instrumentelle Logik in der medialen Funktionalisierung Schwarzer Körper für die Produktion von Bildern des Schiffbruchs, des Überlebenskampfes und des Todes durch Ertrinken: als Repräsentationen der Abjektion bilden sie die Kontrastfolie zur Verkörperung weißer Sozialität und Moralität (vgl. Saucier und Woods 2014, 67).

al. 2017; Taliani 2018) auf die historisch negierte sexuelle und reproduktive Selbstbestimmung Schwarzer Frauen sowie auf die gewaltvolle Verhinderung von Verwandtschafts-, Sorge- und Liebesbeziehungen als eine Dimension sozialen Todes. Eine eingehendere intersektionale Perspektive müsste daher die Dis-/Kontinuitäten zwischen historischen Versklavungs- und gegenwärtigen Grenzregimen herausarbeiten und dabei entlang Schwarzer feministischer Perspektiven (Spillers 1987; Hartman 2016a) die besondere Bedeutung des Schwarzen weiblichen Körpers für gegenwärtige nekropolitische Akkumulations- und neoliberale Fürsorgeregime herausarbeiten (vgl. El-Tayeb und Thompson 2019, 321). Geburt und Mutterschaft vor, während oder nach der traumatisch erlebten Mittelmeerpassage kann dabei auch als eine selbstbestimmte Entscheidung und als eine Form des Widerstands rassifizierter Frauen verstanden werden (Taliani 2018, 114). Zugleich darf die besondere Verletzlichkeit von flüchtenden und geflüchteten Frauen, schwangeren Menschen und geborenen und ungeborenen Kindern auf der Flucht nicht negiert werden. 
Die ästhetische Fetischisierung Schwarzer Menschen, deren Verletzlichkeit bis hin zum Tod zum öffentlichen Spektakel wird, ermöglicht so eine Rezentrierung weißer Handlungsfähigkeit und die Reproduktion weißer Unschuld inmitten eines rassistischen Europa (Dannewid 2017, 1681). ${ }^{14}$ Mit Wekkers Konzept der weißen Unschuld (Wekker 2016) kann die Spektakularisierung Schwarzen Leidens und Sterbens als Konstruktion guter weißer Subjektivität und europäischer Staatsbürger*innenschaft in Abgrenzung zu ihrem schlechten Gegenpart, dem rassistischen und faschistischen Nationalisten, verstanden werden. Durch diese Perspektivierung wird letztlich nicht die Rettung ertrinkender Menschen, sondern die moralische Rettung Europas selbst ermöglicht (Dannewid 2017, 1682). Eine unhinterfragte „Willkommenskultur“ oder eine kosmopolitische Ethik der Hospitalität können dabei geradewegs koloniale Machtasymmetrien reproduzieren, indem Phantasien und Narrative der „Bürde des weißen Mannes“ bzw. der weißen Frau aktualisiert, die konkreten Gewalterfahrungen Schwarzer Menschen ebenso wie ihre politischen Kämpfe und Imaginationen jedoch ausgeblendet werden. Diese Diskursformationen zeigten sich beispielhaft in der Berichterstattung über die Sea-Watch 3 im Sommer 2019. Die Kapitänin Carola Rackete, die schiffbrüchige Geflüchtete aus dem Mittelmeer rettete und trotz des Verbots der italienischen Behörden den Hafen von Lampedusa anlief, wurde als historische Heldin, als Stimme der Vernunft, als Gewissen Europas ikonisiert. Die solidarischen Aktionen unter dem Hashtag \#freecarola, die Demonstrationen für einen Freispruch Racketes und für die Entkriminalisierung der Seenotrettung machten Rackete zur idealisierten Projektionsfläche von europäischen Staatsbürger*innen, die sich solidarisch mit dem Schicksal

14 Die Konsolidierung des moralisch guten weißen Subjekts vollzieht sich dabei auch vor einer postkolonialen Kartographie Europas selbst. Die diskursive Degradierung der südeuropäischen Gesellschaften als faule und unproduktive „Südländer“ insbesondere durch politische und mediale Diskurse in Deutschland wurde zur Rechtfertigung einer harschen Austeritätspolitik als Antwort auf die Eurokrise (Ervedosa 2016). Im Kontext der „Flüchtlingskrise“ dagegen erhalten zivilgesellschaftliche Akteure in Schweden und Deutschland in ihrem Engagement für geflüchtete Menschen weitaus mehr öffentliche Aufmerksamkeit und Anerkennung als Italiener*innen und Griech*innen, die die Geflüchteten unmittelbar beim Überqueren des Mittelmeers und nach ihrer Ankunft auf dem Festland unterstützen und dort die Hauptlast der Solidarität tragen (Bhambra 2017, 398). Zur postkolonialen Dimension der europäischen "Migrationskrise“ in ihrer Verflechtung mit der griechischen Schuldenkrise siehe auch Samaddar (2016). 
flüchtender Menschen zeigten. Gleichzeitig wurden die von ihr geretteten afrikanischen Menschen zur gesichtslosen und passiven Masse, zur „Schiffsladung“, deren unsichtbar gemachte politische Kämpfe keine Solidarität erfahren. Solidarität ist damit ein Privileg derjenigen, deren Menschlichkeit, deren Leben nicht von der Politik rassifizierter Grenzen gefährdet ist und deren Zugehörigkeit nicht infrage gestellt wird: „Solidarity is the product of not being policed not being noticed not having one's humanity called into question fundamentally; belonging is nothing less than the prerogative to ignore the banal terror of policing." (Saucier und Woods 2014, 66)

Die implizite Kolonialität humanitärer Rettung zeigt sich wohl nirgends so plastisch wie in der Benennung der Seenotrettung durch die italienische Küstenwache als Operation „Mare Nostrum“, durch die von Oktober 2013 bis Oktober 2014 nach Angaben der IOM 150.00o Menschenleben gerettet werden konnten. Der historisch-römische Begriffe mare nostrum wurde im italienischen Kolonialkrieg in Libyen 1911 und später im Faschismus unter Mussolini verwendet, um einen italienischen Besitzanspruch auf den gesamten Mittelmeerraum zu erheben und Italienischsein unter kolonialrassistischen und faschistischen Vorzeichen neu zu erfinden (Proglio 2018, 411). Humanitäre Interventionen und solidarische Aktionen, die auf der Levinas'schen Figur der Alterität und der Annahme einer universellen menschlichen Verletzlichkeit beruhen, müssen daher im Kontext des Mittelmeers als nekropolitischer Konstellation auf ihre impliziten Grenzen hin befragt werden. Gegen eine unreflektierte Solidarität, die die Kolonialität der gegenwärtigen Situation im Mittelmeer mit dem Verweis auf \#wirsindallemittendrin oder „We are all on the same boat; and the boat is sinking“ (Bottici 2016) verwässert, gilt es daher, anstelle eines unhinterfragten Postulats der moralischen Gleichheit einer rassistisch unmarkierten Menschheit auf der radikalen Differenz Schwarzen Sterbens im Mittelmeer zu beharren. Diese Differenz kommt in einer Wandmalerei Banksys im als „The Jungle“ bekannten Flüchtlingslager von Calais zum Ausdruck: „We're not all in the same boat“. ${ }^{15}$

15 Banksy (2015), „We're not all in the same boat: Calais, France, 2015“. https:// www.widewalls.ch/murals/banksy-were-not-all-in-the-same-boat; Zugriff: 25. April 2020. 


\section{Das Schwarze Mittelmeer und die Entgrenzung der Demokratie}

Menschen auf der Flucht fordern die Begrenzungen von politischen Einheiten permanent heraus und berühren damit "grundlegende politische Ordnungsfragen" demokratischer Gesellschaften (Schulze Wessel 2017, 210). Sie widersetzen sich dem hegemonialen Blick, der sie als Objekte humanitärer Hilfe taxiert oder aus der Gemeinschaft der Menschheit ausschließt, indem sie selbst als handelnde und politische Subjekte in Erscheinung treten. Sie offenbaren dabei auch den "Nativismus" dominanter theoretischer Perspektiven auf Migration, der in der Frage aufscheint: „What should we do with them?" (kritisch dazu Genova 2016, 345) Doch in welcher Weise sind politische Kämpfe geflüchteter Menschen im Mittelmeer, unter den Bedingungen einer strukturellen rassistischen Verunmöglichung von (Über-)Leben denk- und praktizierbar? Welcher Erfahrungs- und Handlungsraum steht Schwarzen Menschen im Nachleben der Versklavung, in den nekropolitischen Gewaltformationen gegen flüchtende Menschen zur Verfügung? Auch wenn afropessimistisch mit Hartman gedacht naive Vorstellungen von politischer Handlungsfähigkeit und die Romantisierung von widerständigen Subjekten noch im Angesicht extremster Bedingungen der Gewalt und Unterdrückung zurückzuweisen sind, kann das Schwarze Mittelmeer auch als eine Kartographie der politischen Kämpfe verstanden werden, die flüchtige Momente anderer Formen und Praktiken politischer Gemeinschaft jenseits rassistischer Grenzziehungen aufscheinen lässt. Werden in hegemonialen Solidaritätspolitiken vor allem das politische Begehren und die Praxis weiß positionierter Subjekte zentriert und die Stimmen Schwarzer Menschen zum Schweigen gebracht, kann aus der Perspektive des Schwarzen Mittelmeers gefragt werden, mit welchen politischen Deutungen, Imaginationen und Praktiken Schwarze Menschen sich als handelnde Subjekte verstehen und in Bewegung setzen. Geflüchtete und illegalisierte Menschen entwickeln vielfältige Formen der Subversion, der Intervention und der widerständigen Praxis. ${ }^{16}$ Ismail Einashe betont mit Bezug auf das Schwarze Mittelmeer, dass Migrant*innen bei der Überquerung territorialer Grenzen zu politischen Subjekten werden, die auch symbolische Begrenzungen infrage stellen, überschreiten und verschieben: „To illegally cross a border, to

16 Für einen Überblick und eine theoretische Diskussion dieser Praktiken im Lichte französischer politischer Philosophie als radikal-demokratischen Ungehorsams siehe Oberprantacher (2016b). 
evade border guards, to scale metal fences and to survive the Mediterranean Sea is an act not only of survival but of imagination." (Einashe 2018, 23) Am Beispiel der migrantischen Frauenselbstorganisation Women in Exile wird besonders deutlich, wie geflüchtete Schwarze Frauen, die an der Intersektion von anti-Schwarzer und sexualisierter Gewalt stehen, die Widersprüche und Grenzen der europäischen Demokratie offenlegen und demokratische Imaginationen und Praktiken entwickeln, die auf ein Jenseits der existierenden Demokratie in ihren Widersprüchen und Engführungen hinausweisen.

Das Kollektiv Women in Exile geht auf eine Initiative geflüchteter Frauen in Brandenburg im Jahr 2002 zurück, die sich ausgehend von ihrer intersektionalen Verletzlichkeit durch rassistische Gesetzgebungen und sexistische Diskriminierung sowie ihrer Unsichtbarmachung in weißen feministischen Bewegungen einerseits und männlich dominierten Geflüchteteninitiativen andererseits selbst organisierten, um für ihre Rechte zu kämpfen. Women in Exile machen deutlich, dass sie nicht nur auf der Flucht, sondern auch in den Flüchtlingslagern in Deutschland mit der permanenten Gefahr sexualisierter und rassistischer Gewalt konfrontiert sind. Ihre zentrale Forderung, alle Lager zu schließen, wurde im Sommer 2019 erneut in ihrer Dringlichkeit bestätigt. Mitte Juni wurden nahe dem abgelegenen Lager Hohenleipisch in Brandenburg die sterblichen Überreste der 32-jährigen Rita Awour Ojunge gefunden. Die aus Kenia stammende Mutter zweier Kinder lebte dort seit sieben Jahren während ihres noch unabgeschlossenen Asylverfahrens. Seit April 2019 war sie als vermisst gemeldet. Obwohl Freund*innen aus dem Lager mehrfach auf ihr Verschwinden aufmerksam gemacht hatten, wurde erst zwei Monate später eine Suchaktion der Polizei veranlasst. Das Verschwinden Rita Ojunges, ihre mutmaßliche Ermordung inmitten des Waldes, erhielt erst in dem Moment eine größere Öffentlichkeit, nachdem Women in Exile die ihnen zur Verfügung stehenden Ressourcen zur medialen Intervention genutzt hatten. Women in Exile wiesen auch darauf hin, dass die Lagerbewohner*innen seit Jahren durch Proteste für eine Schließung des Lagers kämpfen, da es sich „mitten im Nichts“ befindet und damit kein sicherer Ort für geflüchtete Frauen und Kinder ist, sondern vielmehr ihre Verletzlichkeit erhöht, nicht zuletzt durch männliche Geflüchtete und das Sicherheitspersonal des Lagers (Women in Exile, Flüchtlingsrat Brandenburg 2019). Sie machen damit deutlich, was ein wei $\beta$ positionierter Aktivismus oft übersieht: dass die Zonen anti-Schwarzer Gewalt nicht nur im Mittelmeer zu verorten sind. Die tödlichen Grenzen des Mittelmeers setzen sich mit unverminderter Kraft, als alltägliche Katastrophen im Inneren 
Deutschlands fort. „In Germany, in Bayreuth, Berlin, and Hamburg, the lagers continue. There, refugees are held in villages for years unable to leave without permission, unable to go to the city, unable to find employment or go to school, they are in the living death of the lager." (ebd., 71, meine Hervorhebung)

Die Lager als „Orte der Internierung“ und als „Räume des Ausgrenzens“ machen Menschen zu „Illegalen [...], deren Würde antastbar ist“ (Mbembe 2018). Als Zonen einer kolonialen, auf Rasse beruhenden Nekropolitik markieren sie die inneren Grenzen der liberalen Demokratie, die den rechtlichen, sozialen und körperlichen Tod von rassifizierten Menschen bedeuten oder beschleunigen. Im Gegensatz zu den medial verbreiteten, spektakulären Repräsentationen Schwarzen Sterbens im Mittelmeer als punktuellen Ereignissen tödlicher Gewalt sind die Lager normalisierte Orte eines vorzeitigen Todes, der sich über mehrere Jahre erstreckt, der als „slow violence on refugee bodies“ (vgl. Davies, Isakjee und Dhesi 2017, 1278), als „slow death“ wirkt (ebd., 1280). Women in Exile weisen auf die traumatischen und gesundheitsgefährdenden Haftbedingungen hin, die das Leben Schwarzer Frauen und ihrer Kinder langfristig und dem Blick der Öffentlichkeit entzogen beschädigen und die Bedingungen von Familie und Sozialität Schwarzer Menschen im historischen Schatten des sozialen Todes versklavter Menschen zerstören. Der Tod Rita Ojunges, der hätte verhindert werden können, wurde weder öffentlich bemerkt noch betrauert. Die anti-Schwarze und sexualisierte Gewalt wirkt nicht nur als physische Gewalt im Moment ihrer Ermordung, sondern auch als „violent inaction“ (Davies, Isakjee und Dhesi 2017, 1281) in der Gleichgültigkeit der Polizei, eine Suchaktion einzuleiten und den vorzeitigen Tod einer jungen Frau und Mutter als ein mutmaßliches Gewaltverbrechen einzustufen und aufzuklären. Anti-Schwarze Gewalt verbindet sich hier mit historisch gewachsenen rassifiziert-sexualisierten Konstruktionen Schwarzer Weiblichkeit, die diese entweder negieren oder als bedrohliche Andersartigkeit repräsentieren. Die Frage von Women in Exile, „was wäre passiert, wenn diese Frauen und auch Rita weiße Frauen gewesen wären?“, zielt im Kern darauf ab, wessen Leben als lebenswert und schutzbedürftig gilt und wessen Tod billigend in Kauf genommen wird. Darüber hinaus muss problematisiert werden, warum der Tod Rita Ojunges ebenso wie die Forderungen geflüchteter Frauen nach der Schließung aller Lager in Deutschland nicht einen Bruchteil der Aufmerksamkeit und Solidarität erfahren, die Carola Rackete und der zivilen Seenotrettung im Mittelmeer zuteilwird. Und schließlich, warum die verunmöglichte Mutterschaft 
Rita Ojunges im brandenburgischen Wald nicht denselben Schock hervorruft wie das Bild der im Mittelmeer ertrunkenen Mutter, die noch über die Nabelschnur mit ihrem Kind verbunden ist. Wiederholt sich hier nicht die Dramaturgie eines rassifizierten politischen Imaginären, das Widerstand, Protest und Solidarität mit weißer Subjektivität überschreibt, während Schwarzsein als Objekt und Abjekt außerhalb des Politischen und jenseits der Grenzen einer zu betrauernden Menschheit positioniert wird? ${ }^{17}$

So gesehen kämpfen Women in Exile nicht nur gegen die Inhaftierung geflüchteter Frauen und ihrer Kinder in Lagern an, sondern auch gegen die Einhegung ihrer politischen Praxis im Lagersystem. Wie die Bewohner*innen des Lagers Hohenleipisch in einem offenen Brief nach Rita Ojunges Tod berichten, wird von der Heimleitung und den dort beschäftigten Sozialarbeiter*innen „alles [unternommen], damit wir uns wegen unserer Probleme nicht beschweren, damit das Heim nicht geschlossen wird und sie ihre Arbeit nicht verlieren" (BewohnerInnen des Heims in Hohenleipisch, Brandenburg 2019). Die Versuche der Institution, die widerständigen Praktiken der Bewohner*innen und ihre Kritik an den rassistischen Wirkweisen, Be- und Ausgrenzungen einer auf der Abjektion von Schwarzsein beruhenden demokratischen Gesellschaft zum Schweigen zu bringen, verdeutlichen umso mehr die Potentialität Schwarzer sozialer Bewegungen: Indem sie politische Alternativen zur Kartographie der Grenze und der Logik anti-Schwarzer Gewalt erschließen, werden sie als Gefahr für die bestehende Ordnung wahrgenommen (vgl. Saucier und Woods 2014, 71). Denn Women in Exile problematisieren nicht nur das europäische Grenzregime, sondern die rassistische Konstitution der europäischen Demokratie über ihre grundlegenden Institutionen des Rechts, der Polizei und des Lagers, die in ihrer zentralen Forderung zum Ausdruck kommt: „Our demand is: Women and children out of Lagers! Abolish all Lagers!“ (Kullrich 2017, 219).

17 Hier zeigt sich die Notwendigkeit, unterschiedliche rassistische Geschlechterund Nekropolitiken zu differenzieren. Der postkoloniale „Rettungsdiskurs“, den María do Mar Castro Varela und Nikita Dhawan (2016) in Bezug auf muslimische Frauen in fluchtmigrantischen Communities problematisieren, kann nicht auf Schwarze Frauen in Lagern übertragen werden, deren Gewalterfahrungen weder in den Fokus des öffentlichen Interesses geraten noch als Vorwand für rassistische Diskurse über subalterne Männlichkeiten herangezogen werden. Innerhalb von Grenzregimen und in der Intersektion mit Geschlecht und Klasse hat anti-Schwarzer Rassismus andere Effekte als antimuslimischer Rassismus, unterscheidet sich Abjektion radikal von Subalternität. 
Darin kondensiert sich nicht nur die Kritik am Lager als Ort der Gefangenschaft, des sozialen Todes und der tödlichen Gewalt, sondern auch die politischen Imaginationen einer radikal anderen politischen Ordnung jenseits der rassifizierten und formalen Grenzen der Demokratie (vgl. Smythe 2018, 9). Women in Exile ordnen sich damit in eine lange Tradition eines radikalen Schwarzen Abolitionismus ein, der die Abschaffung von dehumanisierenden und tödlichen Politiken gegen Schwarze Menschen immer in Verbindung mit der Erschaffung neuer Institutionen zur Herstellung materialer Freiheit und Gleichheit denkt. Sie bedienen sich eines Registers widerständiger politischer Imaginationen, das von der gelebten Erfahrung Schwarzer Menschen ausgeht und eine Sprache der Kritik entwickelt, die anders als hegemoniale weiße Gesellschaftskritik - die konstitutive Dimension von Rassismus und anti-Schwarzer Gewalt ausspricht. Ihr Begehren nach einer anderen Demokratie, die in eine abolitionistische Tradition (vgl. Du Bois 1998; Davis 2005; Ehrmann und Thompson 2019) eingeordnet werden kann, imaginiert ein Leben außerhalb des Lagers, eine zukünftige Demokratie ohne Eingesperrtsein, in der Familie, Freund*innenschaft und soziale Beziehungen nicht durch die Kontrolle und Restriktion von Mobilität, durch politische Repression und durch die immanente Gefahr des jederzeit möglichen und straflos bleibenden vorzeitigen Todes Schwarzer Menschen bedroht sind. Ihre politischen Praktiken und Imaginationen eröffnen den Horizont einer demokratischen Praxis, die durch Rasse geschaffene Grenzen überschreitet und die durch Rassismus beschädigte menschliche Relationalität als Grundbedingung demokratischer Gemeinschaften repariert und rehumanisiert.

Women in Exile reihen sich damit in der Kartographie des Schwarzen Mittelmeers in afrikanisch-diasporische Archive der Freiheit ein, die Verhältnisse der Unlebbarkeit mit dem Begehren nach Freiheit und einem guten Leben überschreiben (vgl. Thompson 2018, 246). Ihre Proteste und Interventionen können als Momente einer flüchtigen Demokratie (vgl. Haro und Coles 2019) gefasst werden, die einen politischen Horizont jenseits eines rassifizierten politischen Imaginären aufscheinen lassen. Dazu gehört auch die Frage, wie diejenigen erinnert und betrauert werden können, die ihr Leben im Mittelmeer verloren haben. Im Kontrast zu dominanten Repräsentationen der Gewalt als Spektakel und den Statistiken des Todes, die die verkörperten Erfahrungen Schwarzer Menschen enthistorisieren und entpolitisieren (vgl. Alexander 1994, 92-93), entwickeln Schwarze Künstler*innen Gegenbilder und Gegennarrative, die die ertrunkenen Menschen rehumani- 
sieren und eine Politik des Trauerns ermöglichen. Der Film „Asmat-Names in memory of all victims of the sea“ von Dagmawi Yimer (2014) durchbricht das mathematische Kalkül des Zählens von Körpern. Die Menschen, die am 3. Oktober 2013 bei der Überfahrt nach Europa ertrunken sind, werden noch im Moment tödlicher Gewalt in ihrer körperlichen Integrität gezeichnet und mit ihrem Eigennamen besungen und betrauert. Ihre Namen - darunter Alem (Welt), Selam (Frieden) oder Tesfaye (Hoffnung) - bezeugen ihre politischen Imaginationen jenseits einer vergrenzten Welt, ihre Einbettung in ein menschliches Netz der Sorge, der Verantwortung und der Liebe und die einzigartige Bedeutsamkeit jedes einzelnen Lebens. Yimer schafft es damit, so El-Tayeb, „sowohl die Toten zu ehren als auch Schwarzes Leben zu zelebrieren“ (El-Tayeb und Thompson 2019, 326).

\section{Schwarzes Europa - Jenseits der Grenzen der weißen Demokratie und der politischen Theorie}

Wie ließe sich die Krise der Demokratie in einer Weise denken, die Kolonialität, Rassismus und fortgeführte Nekropolitiken gegen Schwarze Menschen, aber auch deren Kämpfe um die Überwindung der Begrenzungen der Demokratie ernst nimmt? In seinem 1939 erschienenen Buch Black Folk Then and Now identifizierte W. E. B. Du Bois eine verleugnete Vorgeschichte der Krise der Demokratie, wie sie sich im Sieg des Faschismus über Europa manifestierte: die „color line“, die als Rechtfertigung der europäischen Versklavung afrikanischer Menschen geschaffen wurde (Du Bois 2007, 168). Als zentrales Problem des 20. Jahrhunderts organisiert die „color line“ auch nach der Abschaffung der Versklavung nicht nur einen globalen rassifizierten Kapitalismus. Sie beschädigt ebenso nachhaltig die Grundlagen der westlichen liberalen Demokratie (ebd., 264), und zwar in zwei miteinander verbundenen Dimensionen: als „Black Europe, that is, Europe in Africa“ (ebd.), also dem kolonialen Verhältnis zwischen Europa und Afrika, als auch in der Verflechtung von Weißsein und Demokratie. Vor dem Hintergrund aktueller Krisendiagnosen der Demokratie und des immer offener zutage tretenden Rassismus - so auch in den politischen Rufen nach einem „weißen Europa“ (Sheller 2018, 116) - hat Du Bois' in die Zukunft gerichtete Aufforderung, die Probleme der europäischen Demokratie im (post)kolonialen Europa aufzuspüren, unverminderte Aktualität: „An examination of the hard-won fruits of democratic government must involve the relation of white democracy to the colored peoples of the world.“ (ebd., 264) Aus diesem Blickwinkel ist 
das Problem der westeuropäischen liberalen Demokratie nicht eine „Migrationskrise“, sondern die Krise eines europäischen Selbstverständnisses, das Demokratie nur in der rassifizierten Form postkolonialen Weißseins (Genova 2016, 354) zu imaginieren imstande ist. Der Rassismus des Grenzregimes wirkt dabei nicht erst im Moment tödlicher Gewalt. Er ist auch keine Reaktion auf die Präsenz geflüchteter Menschen in Europa, sondern die Europa eingeschriebene Ermöglichungsbedingung einer Politik des Ertrinkenlassen.

Während das „Gespenst des ,illegalen Migranten“ “ im politischen Imaginären Nordamerikas und Europas (vgl. Achiume 2019, 27) Demokratie immer weiter begrenzt und einhegt und das Phantasma eines weißen Europa nährt, stellt die Perspektive des Schwarzen Mittelmeers einen erweiterten Begriffsrahmen für eine Kritik der Grenzen der Demokratie, des Sterbenlassens Schwarzer Menschen im Mittelmeer und der Ambivalenzen solidarischer Bewegungen bereit. Als eine genuin kolonialismus- und rassismuskritische Epistemologie zeigt das Schwarze Mittelmeer zum einen die Leerstellen gegenwärtiger politiktheoretischer Auseinandersetzungen mit Grenzen, Flucht und Migration auf, indem die Kolonialität der europäischen Demokratie und die Rassifizierung ihrer Grenzen offengelegt werden. Wie gezeigt wurde, sind Grenzregime keine bloße Politik der Militarisierung von Grenzen als territorialen Demarkationslinien zwischen Nationalstaaten. Sie manifestieren sich auch als Grenzziehung zwischen einem symbolischen Innen und Außen, zwischen Bürger*in und Nichtbürger*in, Menschen mit und Menschen ohne einklagbare Menschenrechte. Praktiken der Grenzziehung sind daher als Praktiken der Rassifizierung zu verstehen, die festlegen, welche Menschen aus dem Raum des Politischen und letztlich des Menschlichen ausgegrenzt werden. Dieses „Begehren nach Apartheid“, dieser Traum nach einer „reinen“, auf Herkunft und Traditionen gründenden Gemeinschaft ist Mbembe zufolge der sichtbarste Ausweis des Scheiterns der Dekolonisierung Europas, und zwar sowohl in den Beziehungen zwischen Europa und Afrika als auch in Europa selbst (Mensah, Ouendji und Mbembe 2011, 95).

Zum anderen eröffnet das Schwarze Mittelmeer als in sich diasporische Genealogie eines widerständigen Denkens und der politischen Kämpfe Schwarzer Menschen einen Horizont jenseits moralischer und rechtlicher Engführungen von Migration und Flucht sowie nationalstaatlicher Imaginationen der Demokratie, der Impulse für eine Dekolonisierung als Demokratisierung der Demokratie in Europa enthält. Um eine Dekolonisierung der Demokratie denkbar werden zu lassen, ist es jedoch notwendig, eine „politische und ethische Kritik des Rassismus“ (Mbembe 2014, 322) als eine 
bislang vernachlässigte Form der Kritik in die politische Theorie einzuführen. Rassismus bzw. Rasse sind dabei nicht als reine Ideen zu verstehen, die einfach abzulegen oder zu widerlegen wären und als solche im Widerspruch zur Demokratie stehen, sondern als eine Weise des In-der-WeltSeins, die auch in gegenwärtigen Demokratien fortwirkt (Goldberg 2006, 334). Mbembe drängt folglich darauf, eine „radikale Kritik der Rasse in die Demokratie ein[zu]führen" (Mbembe 2014, 318) und diese ausgehend von den politischen Kämpfen Schwarzer Menschen um die „Heraufkunft einer Welt jenseits der Rassen“ (ebd., 322) zu denken. Das Schwarze Mittelmeer in der Tradition einer radikalen Schwarzen Kritik erweist sich hier als eine zentrale Perspektive für die Kritik, Korrektur und Erweiterung einer demokratietheoretischen Perspektive, die geflüchtete Menschen nicht als Träger von Verpflichtungen gegenüber der Aufnahmegesellschaft oder als Objekte humanitärer Hilfe, sondern als politische Akteur*innen zentriert und ihre Kämpfe ebenso ernst nimmt wie die darin enthaltene Kritik der Demokratie.

Zuletzt wirft die Figur des Schwarzen Mittelmeers angesichts der kaum skandalisierten, normalisierten Dimension Schwarzen Sterbens im Mittelmeer mit größerer Dringlichkeit epistemologische sowie forschungsethische Fragen für eine politische Theorie auf, die sich bislang jenseits von Kolonialität als Wissens- und Machtregime der Gegenwart verortet. Wo liegen die kognitiven Grenzen einer „farbenblinden“ politischen Theorie? In welcher Weise überblendet ein normativer Primat von Weißsein deren unhinterfragte universelle Prämissen und Postulate? Warum gelten ausschließlich wei $\beta$ positionierte Theoretiker*innen als autoritativ, um die gelebten Erfahrungen Schwarzer Menschen und von People of Color zu theoretisieren und damit einen epistemischen und immer auch symbolischen und ökonomischen Mehrwert in den Aufmerksamkeitsökonomien hegemonialer, auf rassistischen Ausschlüssen beruhender Wissenschaftsinstitutionen abzuschöpfen? Inwiefern reproduziert die Unsichtbarmachung und theoretische Auslöschung von Schwarzsein, Schwarzer Theoriebildung und Schwarzen Archiven der Freiheit und Demokratie anti-Schwarze Gewalt? Vielsagend ist das bis heute anhaltende Verschweigen Anton Wilhelm Amos in der deutschsprachigen politischen Theorie und Philosophie, dessen aus den Archiven verschwundene Disputation De iure Maurorum in Europa („Über die Rechte Schwarzer Menschen in Europa“) aus dem Jahr 1729 eine unschätzbare Quelle der Kritik eines Schwarzen Europa aus der gelebten Erfahrung eines versklavten „Kammermohren“ und des zugleich ersten Schwarzen Philosophen Deutschlands wäre (Initiative Schwarze Menschen 
in Deutschland 2008, 14-17; Gordon 2008, 35-40; Ette 2014). In Bezug auf diese gewaltvollen Auslöschungen aus einem kolonialen Archiv müsste politische Theorie selbstreflexiv ihre eigenen Begrenzungen transparent machen und problematisieren.

Wie kann politische Theorie mit der Mathematik eines rassistischen Kalküls, den Statistiken des Todes, mit dem Spektakel Schwarzen Sterbens im Fahrwasser der Versklavung umgehen, ohne deren symbolische Gewalt und ihr koloniales Epistem zu reproduzieren (McKittrick 2015a; Sharpe 2016, 50-51)? Statt die Verteilungsfrage zu zentrieren und die Arithmetik Schwarzen Sterbenlassens fortzuführen, ist eine selbstkritische Hinterfragung notwendig, inwiefern euro-nordamerikanische politische Theorie selbst in Ontokolonialität und in die Rassifizierung der Grenzen der Demokratie investiert ist. Die Perspektive des Schwarzen Mittelmeers eröffnet dafür einen erweiterten epistemischen und politischen Horizont. Gegen naive Vorstellungen der Entgrenzung der Demokratie oder die romantische Verklärung von Migrant*innen als das ersehnte revolutionäre Subjekt der Gegenwart könnte die Demokratisierung der Demokratie im Nachleben der Versklavung als die Suche nach einer anderen lebbaren Welt, nach einem anderen demokratischen In-der-Welt-Sein verstanden werden - einer Form des Zusammenlebens, in der wir alle leben können (Hartman, zitiert in $\mathrm{Cu}-$ riel 2019), einer Zukunft, in der Schwarze Leben zählen (Saucier und Woods 2014, 55).

Black Lives Matter.

\section{Literatur}

Achiume, E. Tendayi. 2019. „The Postcolonial Case for Rethinking Borders“. Dissent (Summer 2019). https://www.dissentmagazine.org/article/the-postcolonial-case-for-rethinking-borders. Zugriff: 24. Juli 2019.

Alexander, Elizabeth. 1994 . „Can you be Black and Look at This?‘: Reading the Rodney King Video(s)“. Public Culture 7(1): 77-94. https://doi.org/10.1215/08992363-7$\underline{1-77}$.

Amkpa, Awam. 2016. „Echoes of Pasts, Inscriptions of Presence: ReSignifying the Blackamoors“. In ReSignifications: European Blackamoors, Africana Readings, hg. v. Awam Amkpa, 13-23. Rom: PostcArt.

Arendt, Hannah. 2018. Wir Flüchtlinge. 7. Aufl. Stuttgart: Reclam.

---. 2017. Elemente und Ursprünge totaler Herrschaft: Antisemitismus, Imperialismus, totale Herrschaft. 20. Aufl. München, Berlin, Zürich: Piper. 
Arndt, Susan. 2015. „Racial Turn“. In Wie Rassismus aus Wörtern spricht: (K)Erben des Kolonialismus im Wissensarchiv deutsche Sprache: ein kritisches Nachschlagewerk, hg. von Susan Arndt und Nadja Ofuatey-Alazard. 2. Aufl., 185-189. Münster: Unrast.

Azoulay, Ariella. 2008. The Civil Contract of Photography. New York: Zone Books.

Balibar, Étienne. 2016. „Europe at the Limits“. Interventions. Journal of Postcolonial Studies 18(2): 165-171. https://doi.org/10.1215/08992363-7-1-77.

Balibar, Étienne, Sandro Mezzadra, Eric Fassin und Barbara Spinelli. 2019. „For Justice and Solidarity! Save refugees, stop criminalising sea rescue“. Zugriff: 5 . September 2019. https://you.wemove.eu/campaigns/for-justice-and-solidarity-free-carola-rackete-and-pia-klemp.

Barbara, Giussy, Federica Collini, Cristina Cattaneo, Laura Marasciuolo, Laura Chiappa, Luigi Fedele und Alessandra Kustermann. 2017. „Sexual violence and unwanted pregnancies in migrant women“. The Lancet Global Health 5(4): 396397. https://doi.org/10.1016/S2214-109X(17)30075-X.

Benhabib, Seyla. 2008. Die Rechte der Anderen: Ausländer, Migranten, Bürger. Frankfurt am Main: Suhrkamp.

---. 2016. Kosmopolitismus ohne Illusionen: Menschenrechte in unruhigen Zeiten. Berlin: Suhrkamp.

Benhabib, Seyla, und Slawomir Sierakowski. 2015. Nobody wants to be a refugee: A conversation with Seyla Benhabib. Zugriff am 1. Oktober 2020. https://www. eurozine.com/nobody-wants-to-be-a-refugee/.

BewohnerInnen des Heims in Hohenleipisch, Brandenburg. 2019. „Offener Brief von Bewohner*innen der Flüchtlingsunterkunft in Hohenleipisch“. Pressemitteilung. 17. Juli 2019. Zugriff: 7. September 2019. https://www.fluechtlingsrat-brandenburg.de/aktuelles/offener-brief-von-bewohnerinnen-der-fluechtlingsunterkunft-in-hohenleipisch.

Bhabha, Jacqueline. 2019. Migration als Krise? Wie ein Umdenken möglich ist. Hamburg: Hamburger Edition HIS.

Bhagat, Ali, und Susanne Soederberg. 2019. „Placing Refugees in Authoritarian Neoliberalism: Reflections from Berlin and Paris“. South Atlantic Quarterly 118(2): 421-438. https://doi.org/10.1215/00382876-7381230.

Bhambra, Gurminder K. 2017. „The Current Crisis of Europe: Refugees, Colonialism, and the Limits of Cosmopolitanism“. European Law Journal 23(5): 395-405. Zugriff: 17. Oktober 2018. https://doi.org/10.1111/eulj.12234.

Blumenberg, Hans. 1979. Schiffbruch mit Zuschauer: Paradigma einer Daseinsmethapher. Frankfurt am Main: Suhrkamp.

Bottici, Chiara. 2016. No Border Police, No Border Problems. http://www.publicseminar.org/2016/05/no-border-police-no-border-problems.

Broeck, Sabine. 2018. Gender and the Abjection of Blackness. Albany: State University of New York Press. 
Browne, Simone. 2015. Dark Matters: On the Surveillance of Blackness. Durham: Duke University Press.

Buckel, Sonja. 2018. „Winter is coming. Der Wiederaufbau des europäischen Grenzregimes nach dem ,Sommer der Migration““. PROKLA 48(192): 437-457. https:// doi.org/10.32387/prokla.v48i192.907.

Castro Varela, María do Mar, und Nikita Dhawan. 2016. „Die Migrantin retten!? Zum vertrackten Verhältnis von Geschlechtergewalt, Rassismus und Handlungsmacht“. Österreichische Zeitschrift für Soziologie 41 (S3): 13-28.

Chynoweth, Sarah. 2019. „More Than One Million Pains“: Sexual Violence Against Men and Boys on the Central Mediterranean Route to Italy March. Zugriff: 24. Februar 2021. https://www.womensrefugeecommission.org/research-resources/more-than-one-million-pains-sexual-violence-against-men-and-boys-on-th e-central-mediterranean-route-to-italy/.

Cole, Phillip. 2016. „On the borders of solidarity: Ethics, power and immigration controls“. Soundings: A journal of politics and culture 63(1): 123-135.

Crawley, Heaven, und Dimitris Skleparis. 2018. „Refugees, migrants, neither, both: categorical fetishism and the politics of bounding in Europe's ,migration crisis“". Journal of Ethnic and Migration Studies 44(1): 48-64. https://doi.org/10.1080 $\not 1369183 X .2017 .1348224$.

Curiel, Himeka. 2019. Saidiya Hartman '84 Delivers 2019 Commencement Address. Zugriff: 12. August 2019. https://newsletter.blogs.wesleyan.edu/2019/05/26/ saidiya-hartman-84-delivers-2019-commencement-address/.

Dannewid, Ida. 2017. „White Innocence in the Black Mediterranean: Hospitality and the Erasure of History“. Third World Quarterly 38(7): 1674-1689. https://doi.or $\mathrm{g} / 10.1080 / 01436597.2017 .1331123$.

Davies, Thom, Arshad Isakjee und Surindar Dhesi. 2017. „Violent Inaction: The Necropolitical Experience of Refugees in Europe“. Antipode 49(5): 1263-1284. https://doi.org/10.1111/anti.12325.

Davis, Angela Y. 2005. Abolition Democracy: Beyond Empire, Prisons, and Torture. New York: Seven Stories Press.

Dhawan, Nikita. 2015. The Migrant Crisis and Enlightenment Values: Rescuing the Enlightenment from the Europeans. Zugriff am 9. Oktober 2020. https://www. theeuropean.de/en/nikita-dhawan/10063-the-migrant-crisis-and-enlightenment-values.

Du Bois, W.E.B. 2007. Black Folk Then and Now: An Essay in the History and Sociology of the Negro Race. Hg. v. Henry Louis Gates, Jr. New York: Oxford University Press.

--—. 1998. Black Reconstruction in America. New York: Free Press.

Einashe, Ismail. 2018. „Our Sea: Reading the ,Black Mediterranean“through Europe's Migrant Crisis“. Frieze (199): 23. https://frieze.com/article/reading-black-mediterranean-through-europes-migrant-crisis. Zugriff: 6. August 2019. 
Ehrmann, Jeanette, und Vanessa Eileen Thompson. 2019. „Abolitionistische Demokratie: Intersektionale Konzepte und Praktiken der Strafkritik“. In Strafe und Gefängnis: Theorie, Kritik, Alternativen, hg. von Rehzi Malzahn, 161-181. Stuttgart: Schmetterling.

El-Tayeb, Fatima. 2008. „,The Birth of a European Public': Migration, Postnationality, and Race in the Uniting of Europe“. American Quarterly 60(3): 649-670. https://doi.org/10.1353/aq.0.0024.

El-Tayeb, Fatima, und Vanessa E. Thompson. 2019. „Alltagsrassismus, staatliche Gewalt und koloniale Tradition: Ein Gespräch über Racial Profiling und intersektionale Widerstände in Europa“. In Racial Profiling: Struktureller Rassismus und antirassistischer Widerstand, hg. von Mohamed Wa Baile, Serena O. Dankwa, Tarek Naguib, Patricia Purtschert und Sarah Schilliger, 311-28. Bielefeld: transcript.

Ervedosa, Clara. 2016. „The Calibanisation of the South in the German Public ,Euro Crisis“ Discourse“. Postcolonial Studies: Culture, Politics, Economy 20(2): 137162. https://doi.org/10.1080/13688790.2017.1359873.

Ette, Ottmar. 2014. Anton Wilhelm Amo - Philosophieren ohne festen Wohnsitz: Eine Philosophie der Aufklärung zwischen Europa und Afrika. Berlin: Kadmos.

Farrier, David, und Patricia Tuitt. 2013. „Beyond Biopolitics: Agamben, Asylum, and Postcolonial Critique“. In The Oxford Handbook of Postcolonial Studies, hg. von Graham Huggan. Oxford: Oxford University Press.

Ferreira da Silva, Denise. 2017. „The Banalization of Racial Events“. Theory \& Event 20(1): 61-65.

Fine, Sarah, und Lea Ypi, Hg. 2016. Migration in Political Theory: The Ethics of Movement and Membership. Oxford: Oxford University Press.

Follis, Karolina S. 2015. „Responsibility, Emergency, Blame: Reporting on Migrant Deaths on the Mediterranean in the Council of Europe“. Journal of Human Rights 14(1): 41-62. https://doi.org/10.1080/14754835.2014.987737.

Genova, Nicholas de. 2016. „The ,European‘ Question: Migration, Race, and Post-Coloniality in ,Europe““. In An Anthology of Migration and Social Transformation: European Perspectives, hg. von Anna Amelina, Kenneth Horvath und Bruno Meeus, 343-356. Cham: Springer.

Gilroy, Paul. 1993. The Black Atlantic: Modernity and Double Consciousness. Cambridge, Mass. Harvard University Press.

- - . 2004. „Migrancy, Culture, and a New Map of Europe“. In Blackening Europe: The African American Presence, hg. v. Heike Raphael-Hernandez, xi-xxi. New York: Routledge.

- - . 2018. „,Where every breeze speaks of courage and liberty': Offshore Humanism and Marine Xenology, or, Racism and the Problem of Critique at Sea Level“. Antipode 50(1): 3-22. https://doi.org/10.1111/anti.12333.

Goldberg, David Theo. 2006. „Racial Europeanization“. Ethnic and Racial Studies 29(2): 331-64. https://doi.org/10.1080/01419870500465611 
Gordon, Lewis R. 2008. An Introduction to Africana Philosophy. Cambridge: Cambridge University Press.

Gregory, Derek. 2004. The Colonial Present: Afghanistan, Palestine, Iraq. Oxford: Blackwell.

Grovogui, Siba N. 2002. „Regimes of Sovereignty: International Morality and the African Condition“. European Journal of International Relations 8(3): 315-338. https://doi.org/10.1177/1354066102008003001.

Gündoğdu, Ayten. 2015. Rightlessness in an Age of Rights: Hannah Arendt and the Contemporary Struggles of Migrants. New York: Oxford University Press.

Gutiérrez Rodríguez, Encarnación. 2010. „Decolonizing Postcolonial Rhetoric“. In Decolonizing European Sociology: Transdisciplinary Approaches, hg. v. Encarnación Gutiérrez Rodríguez, Manuela Boatca, Sérgio Costa und Robert Holton, 49-67. Farnham: Taylor \& Francis.

- - . 2018. ,The Coloniality of Migration and the ,Refugee Crisis': On the Asylum-Migration Nexus, the Transatlantic White European Settler Colonialism-Migration and Racial Capitalism“. Refuge 34(1): 16-28. https://doi.org/10.7202/1050851.

Ha, Kien Nghi. 2003. „Die kolonialen Muster deutscher Arbeitsmigrationspolitik“. In Spricht die Subalterne deutsch? Migration und postkoloniale Kritik, hg. von Encarnación Gutiérrez Rodríguez und Hito Steyerl, 56-107. Münster: Unrast.

Habermas, Jürgen. 2018. „Wo bleibt die proeuropäische Linke?“. Blätter für deutsche und internationale Politik (12): 41-46.

Hanchard, Michael. 2010. „Contours of Black Political Thought: An Introduction and Perspective“. Political Theory 38(4): 510-536. https://doi. org/10.1177/o090591710366379.

Hardin, Garrett. 1974. „Commentary: Living on a Lifeboat“. BioScience 24(10): 561568. https://doi.org/10.2307/1296629

Haro, Lia, und Romand Coles. 2019. „Reimagining Fugitive Democracy and Transformative Sanctuary with Black Frontline Communities in the Underground Railroad“. Political Theory, 1-28. https://doi.org/10.1177/0090591719828725.

Hart, William David. 2018. „Constellations: Capitalism, Antiblackness, Afro-Pessimism, and Black Optimism“. American Journal of Theology \& Philosophy 39(1): 5-33.

Hartman, Saidiya. 1997. Scenes of Subjection: Terror, Slavery, and Self-Making in Nineteenth-Century America. New York: Oxford University Press.

---. 2007. Lose Your Mother: A Journey Along the Atlantic Slave Route. New York: Farrar, Straus and Giroux.

- - . 2016a. „The Belly of the World: A Note on Black Women's Labors“. Souls. A Critical Journal of Black Politics, Culture, and Society 18(1): 166-173. https:// doi.org/10.1080/10999949.2016.1162596

- - . 2016b. „The Dead Book Revisited“. History of the Present 6(2): 208-216. https://doi.org/10.5406/historypresent.6.2.0208. 
Hartman, Saidiya, und Frank B. Wilderson. 2003. „The Position of the Unthought“. Qui Parle 13(2): 183-201. https://doi.org/10.1215/quiparle.13.2.183.

Heller, Charles, Lorenzo Pezzani und Situ Research. 2014. „Case: ,Left-to-Die Boat““. In FORENSIS: The Architecture of Public Truth, hg. v. Eyal Weizman, Anselm Franke und Forensic Architecture, 637-655. Berlin, New York: Sternberg Press.

Hess, Sabine. 2018. Es reicht! Wenn Unrecht zur Norm wird. Zugriff am 9. Oktober 2020. http://kritnet.org/2018/es-reicht-wenn-unrecht-zur-norm-wird/.

Hess, Sabine, Bernd Kasparek, Stefanie Kron, Mathias Rodatz, Maria Schwertl und Simon Sontowski. 2017. „Der Lange Sommer Der Migration: Krise, Rekonstitution Und Ungewisse Zukunft Des Europäischen Grenzregimes“. In Der Lange Sommer Der Migration: Grenzregime III, hg. von Sabine Hess, Bernd Kasparek, Stefanie Kron, Mathias Rodatz, Maria Schwertl und Simon Sontowski. 2. korrigierte Auflage, 6-24. Berlin, Hamburg: Assoziation A.

Hesse, Barnor. 2007. „Racialized modernity: An analytics of white mythologies“. Ethnic and Racial Studies 30(4): 643-663. https://doi. org/10.1080/01419870701356064.

-_- 2009. „Black Europe's Undecidability“. In Black Europe and the African Diaspora, hg. v. Darlene C. Hine, Trica D. Keaton und Stephen Small, 291-304. Urbana: University of Illinois Press.

hooks, bell. 1992. „Representing Whiteness in the Black Imagination“. In Cultural Studies, hg. v. Lawrence Grossberg, Cary Nelson und Paula Treichler, 338-346. New York: Routledge.

Initiative Schwarze Menschen in Deutschland, Hg. 2008. Homestory Deutschland: Schwarze Biografien in Geschichte und Gegenwart. Bonn: Bundeszentrale für politische Bildung.

IOM. 2019. Missing Migrants Project: Tracking Deaths Along Migratory Routes. Zugriff: 1. August 2019. https://missingmigrants.iom.int/region/mediterranean.

Ivison, Duncan. 2002. Postcolonial Liberalism. Cambridge, UK, New York: Cambridge University Press.

James, Joy. 2016. „The Womb of Western Theory: Trauma, Time Theft, and the Captive Maternal“. Carceral Notebooks 12: 253-296.

Kelley, Robin D.G. 2000. „Foreword“. In Black Marxism: The Making of the Black Radical Tradition, xi-xxxiii. Chapel Hill: University of North Carolina Press.

Kerner, Ina. 2019. „Grenzen und Migration. Postkoloniale Perspektiven“. ZfP Zeitschrift für Politik 66(2): 199-216. https://doi.org/10.5771/0044-3360-2019-2199.

Kukathas, Chandran. 2016. „Are Refugees Special?“. In Migration in Political Theory: The Ethics of Movement and Membership, hg. v. Sarah Fine und Lea Ypi, 249-268. Oxford: Oxford University Press. 
Kullrich, Nina. 2017. „,A Bridge between the Refugee Movements and the Feminist Movements'. Interview with Bethi Ngari from Women in Exile“. Movements. Journal for Critical Migration and Border Regime Studies 3(1): 217-222.

Lauré al-Samarai, Nicola. 2005. „Inspirited Topography: Über/Lebensräume, Heim-Suchungen und die Verortung der Erfahrung in Schwarzen deutschen Kultur- und Wissenstraditionen“. In Mythen, Masken Und Subjekte: Kritische Weißseinsforschung in Deutschland, hg. von Maureen M. Eggers, Grada Kilomba, Peggy Piesche und Susan Arndt, 118-134. Münster: Unrast.

Lentin, Alana. 2008. „Europe and the Silence about Race“. European Journal of Social Theory 11(4): 487-503. https://doi.org/10.1177/1368431008097008.

Linke, Uli. 2011. „Technologies of Othering: Black Masculinities in the Carceral Zones of European Whiteness". In Europe in Black and White: Immigration, Race and Identity in the ,Old Continent', hg. v. Manuela R. Sanches, Fernando Clara, João F. Duarte und Leonor P. Martins, 123-141. Chicago: Intellect.

Lobenstein, Caterina, und Mariam Lau. 2018. Seenotrettung: Oder soll man es lassen? Zugriff: 1. August 2019. https://www.zeit.de/2018/29/seenotrettung-fluechtlinge-privat-mittelmeer-pro-contra.

Maio, Alessandra di. 2014. „Lampedusa und das ,Schwarze Mittelmeer‘. Die Migrationsrouten im Jahrtausend der Globalisierung“. Zibaldone. Zeitschrift für italienische Kultur der Gegenwart, 17-31.

Mavelli, Luca. 2017. „Governing populations through the humanitarian government of refugees: Biopolitical care and racism in the European refugee crisis“. Review of International Studies 43(5): 809-832. https://doi.org/10.1017/ $\underline{\text { So260210517000110. }}$.

Mayblin, Lucy. 2014. „Colonialism, Decolonisation, and the Right to be Human: Britain and the 1951 Geneva Convention on the Status of Refugees“. Journal of Historical Sociology 27(3): 423-441. https://doi.org/10.1111/johs.12053.

Mbembe, Achille. 2003. „Necropolitics“. Public Culture 15(1): 11-40. https://doi. org/10.1215/08992363-15-1-11.

---. 2014. Kritik der Schwarzen Vernunft. Berlin: Suhrkamp.

---. 2018. Die große Vergrenzung. Zugriff am 2. Oktober 2020. https://taz.de/ Sicherheit-und-Migration/!5517629/.

McKittrick, Katherine. 2015a. „Mathematics Black Life“. The Black Scholar 44(2): 16-28. https://doi.org/10.1080/00064246.2014.11413684.

- - . 2015b. „Yours is the Intellectual Struggle: Sylvia Wynter and the Realization of the Living“. In Sylvia Wynter: On Being Human as Praxis, hg. v. Katherine McKittrick, 1-8. Durham: Duke University Press.

Menke, Christoph. 2016. „Zurück zu Hannah Arendt - die Flüchtlinge und die Krise der Menschenrechte“. Merkur. Deutsche Zeitschrift für europäisches Denken, 70(806): 49-58. 
Mensah, Ayoko, Norbert N. Ouendji und Achille Mbembe. 2011. „Le temps de l'Afrique viendra“. Africultures 83(1): 87-100. https://doi.org/10.3917/afcul.083.0087.

Mezzadra, Sandro, und Brett Neilson. 2013. Border as Method, or, the Multiplication of Labor. Durham: Duke University Press.

Miller, David. 2016. Strangers in our midst: The political philosophy of immigration. Cambridge, London: Harvard University Press.

Mills, Charles W. 1994. „Revisionist Ontologies: Theorizing White Supremacy“. Social and Economic Studies 43(3): 105-134.

-- - 2007. „White Ignorance“. In Race and Epistemologies of Ignorance, hg. v. Shannon Sullivan und Nancy Tuana, 11-38. Albany: State University of New York Press.

-_-. 2017. „Criticizing Critical Theory“. In Critical Theory in Critical Times: Transforming the Global Political and Economic Order, hg. v. Penelope Deutscher und Cristina Lafont, 233-250. New York: Columbia University Press.

Oberprantacher, Andreas. 2016a. „The Circulation of Camps in the Peripheries and the Desire for Undesirables“. Lo Squaderno (41): 39-41.

- - . 2016b. „Radikal demokratischer Ungehorsam. ,Illegale“ als strittiges politisches Subjekt“. Zeitschrift für Praktische Philosophie 3(1): 305-338. https://doi. org/10.22613/zfpp/3.1.10.

Pasha, Mustapha Kamal. 2011. „Untimely reflections“. In International Relations and Non-Western Thought: Imperialism, Colonialism, and Investigations of Global Modernity, hg. v. Robbie Shilliam, 217-226. New York: Routledge.

Peabody, Sue. 1997. „The Police des Noirs, 1776-1777“. In „There Are No Slaves in France“: The Political Culture of Race and Slavery in the Ancien Régime, hg. v. Sue Peabody, 106-120. New York, Oxford: Oxford University Press.

phoenix. 2019. „Prof. Sonja Buckel (Politikwissenschaftlerin) zur Flüchtlingsthematik“. 3. Juli 2019. phoenix vor Ort. https://www.youtube.com/watch?v=p-Ldo68fk1U\&feature=youtu.be.

Pichl, Maximilian, und Timo Tohidipur. 2019. „Verpflichtung zur aktiven Seenotrettung: $\mathrm{Zu}$ den nicht intendierten Effekten der Überwachung des Mittelmeers durch Frontex“. In An den Grenzen Europas und des Rechts, hg. v. dens., 177208. Bielefeld: transcript.

Proglio, Gabriele. 2018. „Is the Mediterranean a White Italian-European Sea? The multiplication of borders in the production of historical subjectivity“. Interventions 20(3): 406-427. https://doi:10.1080/1369801X.2017.1421025.

Quijano, Aníbal. 2019. Kolonialität der Macht, Eurozentrismus und Lateinamerika. Wien, Berlin: Turia + Kant.

Rediker, Marcus. 2007. The Slave Ship: A Human History. London: John Murray.

Robinson, Cedric J. 2000. Black Marxism: The Making of the Black Radical Tradition. Chapel Hill: University of North Carolina Press. 
Rothberg, Michael. 2019. The Implicated Subject: Beyond Victims and Perpetrators. Stanford: Stanford University Press.

Sager, Alex. 2014. „Methodological Nationalism, Migration and Political Theory“. Political Studies 64(1): 42-59. https://doi.org/10.1111/1467-9248.12167.

Samaddar, Ranabir. 2016. A Post-Colonial Enquiry into Europe's Debt and Migration Crisis. Singapore: Springer.

Sanyal, Debarati. 2017. „Calais's ,Jungle': Refugees, Biopolitics and the Arts of Resistance“. Representations (139): 1-22. https://doi.org/10.1525/rep.2017.139.1.1.

Saucier, P. Khalil, und Tryon W. Woods. 2014. „Ex Aqua: The Mediterranean Basin, Africans on the Move and the Politics of Policing“. Theoria: A Journal of Social and Political Theory 61(141): 55-75. https://doi.org/10.3167/th.2014.6114104.

Schulze Wessel, Julia. 2017. Grenzfiguren - Zur politischen Theorie des Flüchtlings. Bielefeld: transcript.

Scott, D. 2013. „On the Very Idea of a Black Radical Tradition“. Small Axe: A Caribbean Journal of Criticism 17(1 40): 1-6. https://doi.org/10.1215/079905371665398.

Sexton, Jared. 2016. „Afro-Pessimism: The Unclear Word“. Rhizomes: Cultural Studies in Emerging Knowledge, Nr. 29: 1-35. https://doi.org/10.20415/rhiz/o29. e01.

Sharpe, Christina. 2016. In the Wake: On Blackness and Being. Durham: Duke University Press.

Sheller, Mimi. 2018. Mobility Justice: The Politics of Movement in an Age of Extremes. London, Brooklyn: Verso.

Slobodian, Quinn. 2019. „No More Lifeboats“. Dissent Summer 2019. https://www. dissentmagazine.org/article/no-more-lifeboats. Zugriff: 25. Juli 2019.

Smythe, SA. 2018. „The Black Mediterranean and the Politics of Imagination“. Middle East Report (286): 3-9.

Spillers, Hortense J. 1987. „Mama's Baby, Papa's Maybe: An American Grammar Book“. Diacritics 17(2): 64. https://doi.org/10.2307/464747.

Spivak, Gayatri Chakravorty. 2013. An Aesthetic Education in the Era of Globalization. Cambridge, London: Harvard University Press.

Steinhilper, Elias, und Rob Gruijters. 2017. Border Deaths in the Mediterranean: What We Can Learn from the Latest Data. Zugriff: 1. August 2019. https://www. law.ox.ac.uk/research-subject-groups/centre-criminology/centreborder-criminologies/blog/2017/03/border-deaths.

Taliani, Simona. 2018. „Sometimes I Feel Like a Motherless Child: Nigerian Migration, Race Memories and the Decolonization of Motherhood“. In Migration and the Contemporary Mediterranean: Shifting Cultures in Twenty-First-Century Italy and Beyond, hg. von Claudia Gualtieri, 111-130. Oxford: Peter Lang. 
Thompson, Vanessa E. 2018. „Repairing Worlds: On Radical Openness beyond Fugitivity and the Politics of Care: Comments on David Goldberg's Conversation with Achille Mbembe“. Theory, Culture \& Society 35(7-8): 243-250. https://doi. org/10.1177/0263276418808880.

Trouillot, Michel-Rolph. 2002. „North Atlantic Universals: Analytical Fictions, 1492-1945“. South Atlantic Quarterly 101(4): 839-858. https://doi. org/10.1215/00382876-101-4-839.

UNITED Against Refugee Deaths. 2019. Fatal Policies of Fortress Europe. Pressemitteilung. 20. Juni 2019. Zugriff: 26. Juli 2019. http://www.unitedagainstracism. org/blog/2019/06/20/press-release-international-refugee-day-20th-june-2019.

United Nations High Commissioner for Refugees. 2018. 2,0oo Lives and Counting: Mediterranean Death Toll in 2018. Pressemitteilung. 6. November 2018. Zugriff: 26. Juli 2019. https://www.unhcr.org/news/briefing/2018/11/ 5be15cf34/200o-lives-counting-mediterranean-death-toll-2018.html.

Vacano, Diego A. von. 2012. The Color of Citizenship: Race, Modernity and Latin American/Hispanic Political Thought. Oxford: Oxford University Press.

Weheliye, Alexander G. 2014. Habeas Viscus: Racializing Assemblages, Biopolitics, and Black Feminist Theories of the Human. Durham: Duke University Press.

Wekker, Gloria. 2009. „Another Dream of a Common Language: Imagining Black Europe“. In Black Europe and the African Diaspora, hg. v. Darlene C. Hine, Trica D. Keaton und Stephen Small, 2777-2289. Urbana: University of Illinois Press.

-_-. 2016. White Innocence: Paradoxes of Colonialism and Race. Durham: Duke University Press.

Wilderson, Frank B. 2010. Red, White \& Black: Cinema and the Structure of U.S. Antagonisms. Durham: Duke University Press.

Women in Exile. 2019. Desolate Situation nach ungeklärtem Tötungsdelikt in Flüchtlingsunterkunft: „Wir wollen einfach nur weg von hier“. 2019. Pressemitteilung. 23. Juli 2019. Zugriff: 7. September 2019. https://www.women-in-exile. net/desolate-situation-nach-ungeklaertem-toetungsdelikt-in-fluechtlingsunterkunft-wir-wollen-einfach-nur-weg-von-hier/.

Wynter, Sylvia. 1994. „No Humans Involved“. An Open Letter to my Colleagues“. Forum N.H.I. Knowledge for the 21st Century 1(1): 42-73.

- - . 2003. „Unsettling the Coloniality of Being/Power/Truth/Freedom: Towards the Human, After Man, Its Overrepresentation - An Argument“. CR: The New Centennial Review 3(3): 257-337. https://doi.org/10.1353/ncr.2004.0015.

Yimer, Dagmawi. 2014. ASMAT-Names in memory of all victims of the sea. Zugriff am 16. Oktober 2020. https://vimeo.com/114343040. 